\title{
Specificity in the Axonal Connections of Layer VI Neurons in Tree Shrew Striate Cortex: Evidence for Distinct Granular and Supragranular Systems
}

\author{
W. Martin Usrey and David Fitzpatrick \\ Department of Neurobiology, Duke University Medical Center, Durham, North Carolina 27710
}

Pyramidal neurons in layer $\mathrm{VI}$ of striate cortex are the source of descending projections to the lateral geniculate nucleus (LGN) as well as a major source of axon terminals in the layers of striate cortex that receive LGN projections. This study examines how the connections of layer VI neurons are arranged with respect to the functionally distinct classes of neurons that compose their cortical and subcortical targets. By placing injections of biocytin into layer Vl of tree shrew striate cortex, we identified two sublayers that differ in their intracortical and thalamic connections. Neurons in the upper part of layer VI, layer Vla, terminate in cortical layer IV, whereas those in the lower part of layer $\mathrm{VI}$, layer $\mathrm{VIb}$, terminate throughout the supragranular layers, layers I-III. The selectivity of layer VI subdivisions for the granular and supragranular layers is also evident in their descending projections. Neurons in layer Vla terminate preferentially in the LGN layers that supply layer IV (LGN layers $1,2,4$, and 5), whereas neurons in layer VIb terminate in the LGN layers that supply layers I-III (LGN layers 3 and 6) and in the pulvinar nucleus. Additional subclasses of layer Vla neurons were identified based on the restriction of their terminal fields to narrow subtiers within layer IV. By influencing the activity of distinct populations of cortical neurons and the thalamic neurons that supply them, layer VI neurons could exert a powerful influence on the flow of activity in functionally distinct cortical circuits.

Key words: visual cortex; area 17; lateral geniculate nucleus; pulvinar; corticothalamic pathways; ON, OFF pathways
Pyramidal neurons in layer VI of striate cortex are the source of descending projections to the lateral geniculate nucleus (LGN) as well as a major source of axon terminals in layer IV of striate cortex-the layer that receives most of the terminals from LGN relay cells (Gilbert and Kelly, 1975; Lund and Boothe, 1975; Lund et al., 1975; Hendrickson et al., 1978; Gilbert and Wiesel, 1979; Fitzpatrick et al., 1985; Katz, 1987). A striking feature of both target regions is a diversity of cell types as defined by morpholugical and physiological criteria. For example, LGN relay cells differ in their size, the temporal and spatial aspects of their visual responses, and the sign of their response (ON- or OFF-center)differences that reflect, to a large extent, the properties of their ganglion cell inputs (Cleland et al., 1971; Dreher et al., 1976; Sherman et al., 1976; Wilson et al., 1976; Schiller and Malpeli, 1978; Kaplan and Shapley, 1982; Sherman, 1985; Shapley and Perry, 1986). A comparable diversity is present within cortical layer IV, due in part to specificity in the termination patterns of different classes of LGN neurons (Hubel and Wiesel, 1972; Blasdel and Lund, 1983; Humphrey et al., 1985; Raczkowski and Fitzpatrick, 1990). This study examines how the connections of layer VI neurons are arranged with respect to the functionally distinct classes of neurons that compose their cortical and subcortical targets.

Received June 20, 1995; revised Oct. 16, 1995; accepted Nov. 6, 1995.

This work was supported by National Institutes of Health Grant EY06821 and a Sigma Xi Grants in Aid of Research award. We thank Martha Foster for her expert technical assistance and Nell Cant, William Hall, and Brett Schofield for insightful comments on this manuscript.

Correspondence should be addressed to David Fitzpatrick, Department of Neurobiology, P.O. Box 3209, Duke University Medical Center, Durham, NC 27710.

Dr. Usrey's present address: Department of Neurobiology, Goldenson Building, Harvard Medical School, Boston, MA 02115.

Copyright 1996 Society for Neuroscience 0270-6474/96/161203-16\$05.00/0
Studies in primates, in which the clear separation of cell types into layers makes this issue easier to approach, have raised the possibility of a simple rule that relates the patterns of intracortical and thalamic connections: individual layer VI neurons synapse with a limited set of layer IV neurons and with the LGN neurons that supply them (Lund and Boothe, 1975; Lund et al., 1975). Evidence for the matching of local and distant connections comes from considering the relation of layer VI connections to the parvo- and magnocellular streams (Schiller and Malpeli, 1978; Schiller and Logothetis, 1990). Neurons in the upper part of macaque layer VI project to the parvocellular layers of the LGN and to cortical layers IVC $\beta$ and IVA, the layers that receive terminals from parvocellular LGN neurons; in contrast, neurons in the lower part of layer VI project to the magnocellular layers of the LGN and to layer IVC $\alpha$, the target of magnocellular axon terminals (Lund and Boothe, 1975; Lund et al., 1975).

These results, however, leave open the question of whether the correspondence in local and distant connections can be extended to other types of lateral geniculate relay cells. One class of relay cell that has been overlooked in considering subtypes of layer VI neurons is the population of small, pale-staining neurons that comprise the intercalated layers (Fitzpatrick et al., 1983; Lachica and Casagrande, 1992; Hendry and Yoshioko, 1994). In addition to receiving a rich descending projection from layer VI, these neurons present an interesting challenge to the proposed rule; unlike their counterparts in the principal layers, the axons of intercalated layer neurons pass through layer IV to terminate in the supragranular layers (layers I-III) (Fitzpatrick et al., 1983; Conley et al., 1984; Lachica and Casagrande, 1992; Usrey et al., 1992; Hendry and Yoshioko, 1994). A projection from layer VI to the supragranular layers has been described in rat visual cortex (Burkhalter, 1989); however, it remains to be seen whether this 


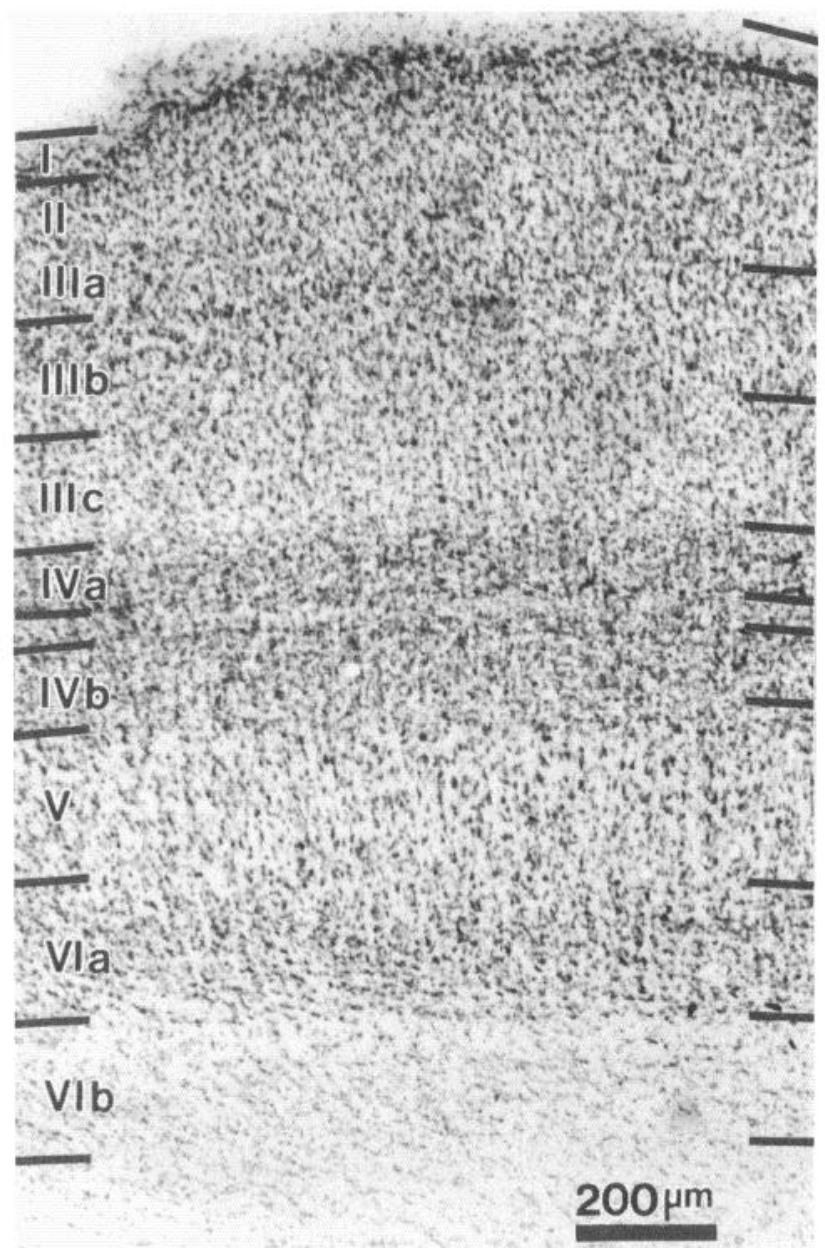

Figure 1. Photomicrograph of a Nissl-stained section of striate cortex Laver IV is subdivided by a cell-sparse cleft into $I V a$ and $I V b$. Layer III is subdivided based on cell density into three sublayers: IIIa, IIIb, and IIIc Layer VI is subdivided based on cell density into two sublayers: VIa and $V I b$.

projection is present in other species and, if so, whether the neurons giving rise to this projection also project to the LGN.

It is also unclear how layer VI projections are organized with respect to the ON- and OFF-center subtypes of the LGN relay cells that terminate in layer IV. In several species, these $\mathrm{ON}-$ and OFF-center subtypes are segregated into different LGN layers (Schiller and Malpeli, 1978; LeVay and McConnell, 1982; Conway and Schiller, 1983; Stryker and Zahs, 1983; Schiller, 1992), an arrangement that would seem ideal for ensuring that they sample from different sets of layer VI inputs. But, whether the terminal fields of individual layer VI neurons are actually selective for the $\mathrm{ON}$ - versus OFF-center layers of the LGN remains unknown.

We hoped to gain insight into these issues by using microinjections of biocytin to examine the local and long-distance connections of layer VI neurons in striate cortex of the tree shrew. As with primates, tree shrews display a highly laminated geniculostriate system, with separate sets of LGN layers providing inputs to layer IV and the supragranular layers (Carey et al., 1979; Conley et al., 1984; Usrey et al., 1992). Furthermore, ON and OFF classes of retinal ganglion cells terminate in separate LGN layers, and these layers in turn terminate in different subtiers of layer IV (Harting et al., 1973; Conway and Schiller, 1983; Conley et al., 1984; Raczkowski and Fitzpatrick, 1990). This arrangement
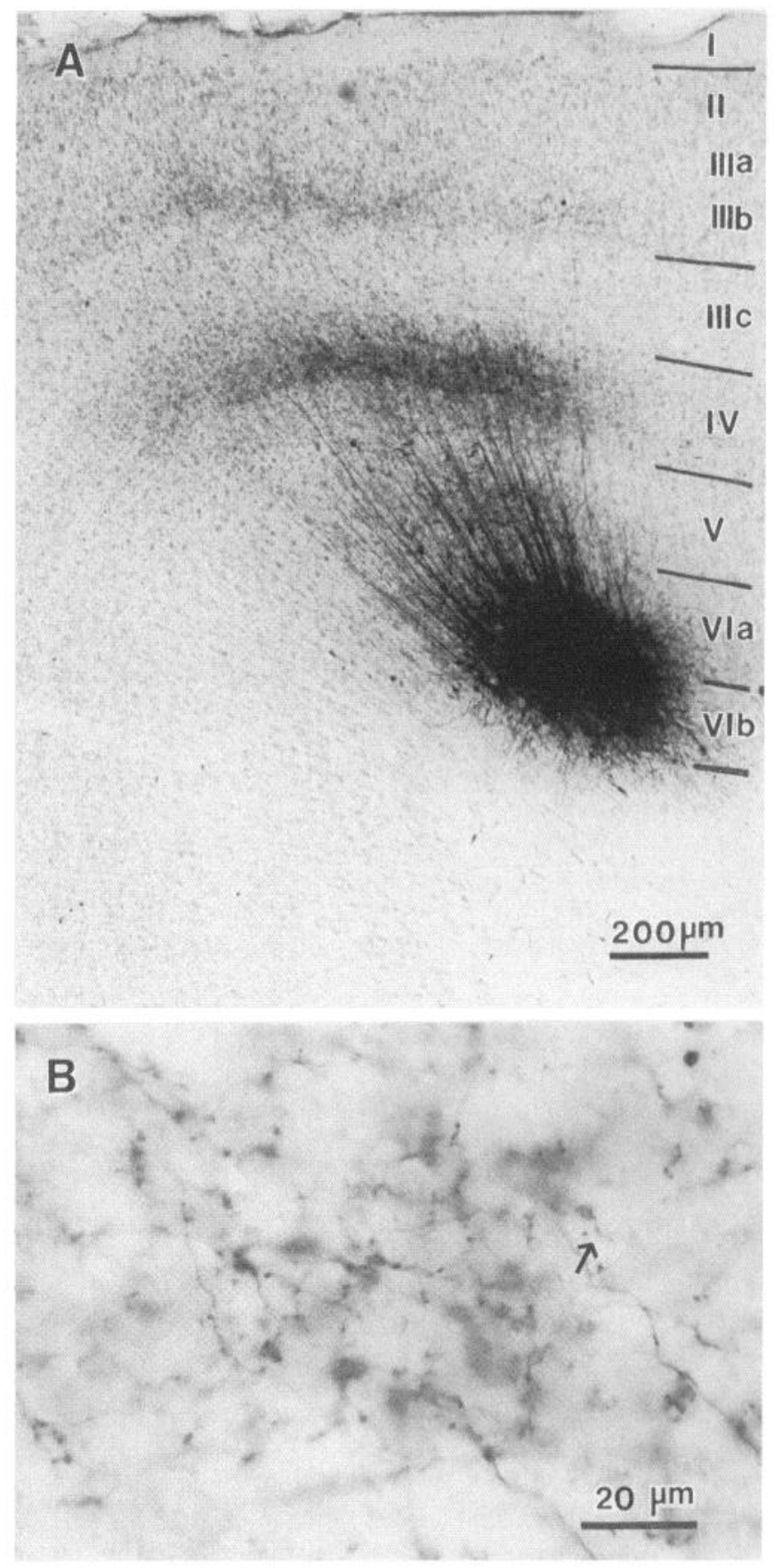

Figure 2. An example of a biocytin injection site in layer VI of striate cortex and the resulting pattern of labeled axons and terminals in the overlying cortical layers. $A$, Photomicrograph of a Nissl-stained section of striate cortex with an injection of biocytin centered at the layer $V I a-V I b$ border. Above the injection site, labeled fibers and terminals can be seen both in layer $I V$ and in layer $I I I b$. B. High-power photomicrograph of layer IV revealing the fine nature of the axons and boutons. Most of the boutons are attached to the axons by short, thin stalks (arrow).

provides a unique opportunity to assess the relation of layer VI neurons to the ON and OFF classes of relay cells and their cortical targets.

\section{MATERIALS AND METHODS}

Animal preparation. Eighteen adult tree shrews, Tupaia belangeri, of both sexes were used in this study. Surgical anesthesia was induced with a mixture of ketamine $(180 \mathrm{mg} / \mathrm{kg})$ and xylazine $(5.75 \mathrm{mg} / \mathrm{kg})$ and main- 
A

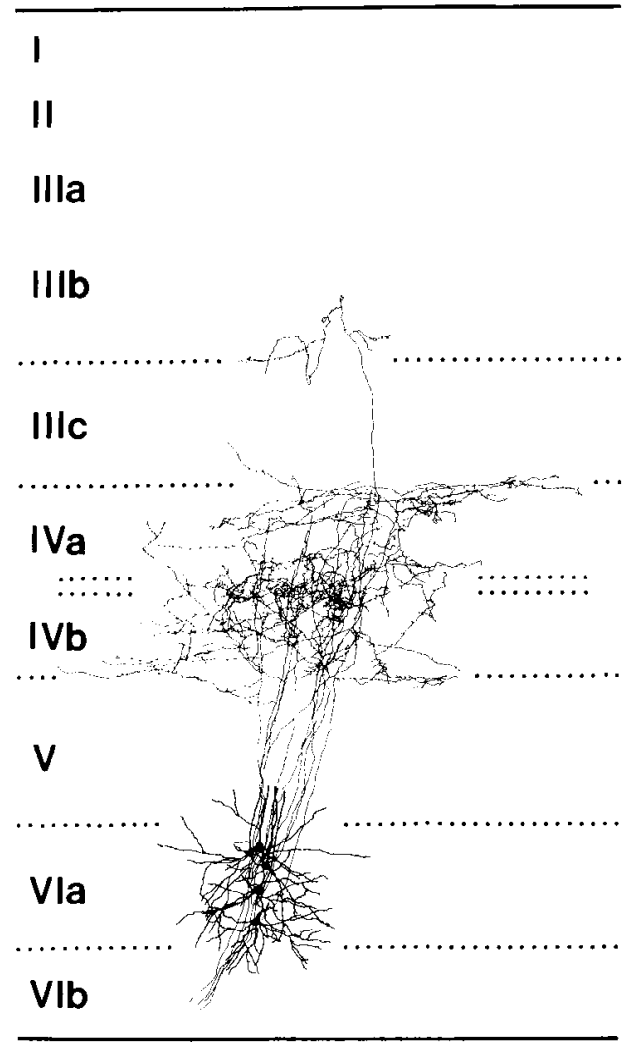

B

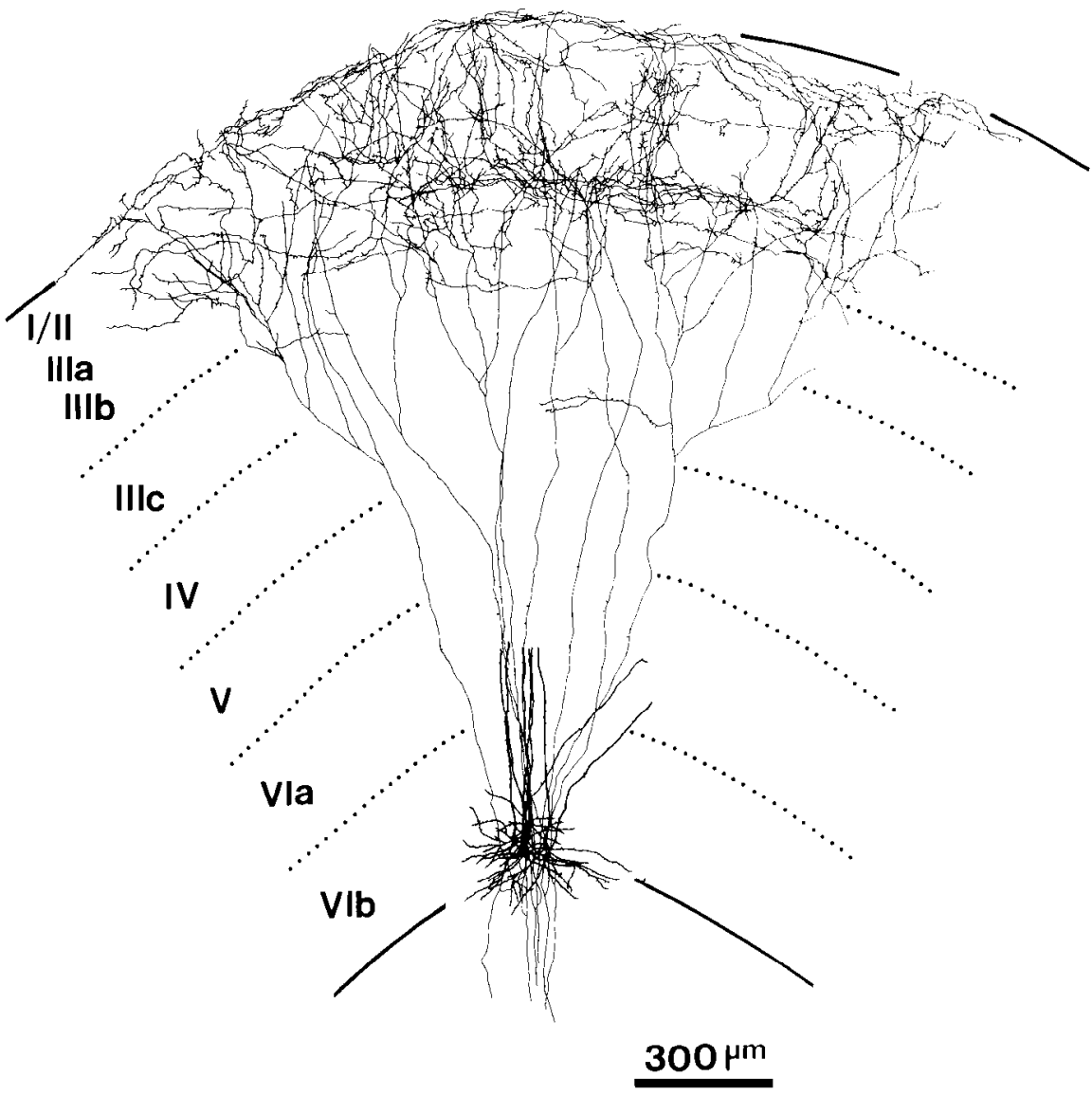

Figure 3. Camera lucida drawings of the intrinsic projections from small populations of neurons in layer VIa and VIb of striate cortex labeled by injections of biocytin. $A$, Axons from a population of layer $V I a$ neurons arborize extensively across the depth of layer $I V$. A single collateral extends above the layer IV terminal field into layer $I I l b$. $B$, Axons from a population of layer $V I b$ neurons arborize extensively in layers $I I I b-I$. Layer $I V$ is void of terminals except for those on a short branch of one of the ascending axons. All of the neurons in $A$ and $B$ are pyramids; however, apical dendrites were not reconstructed above layer VI to prevent obscuring the axon terminal fields.

tained with supplements of $0.1-0.2 \mathrm{ml}$ of sodium pentobarbital $(10$ $\mathrm{mg} / \mathrm{ml}$, i.p.). Body temperature was maintained at $37^{\circ} \mathrm{C}$ using a thermostatically controlled heating blanket. Animals were placed in a stereotaxic apparatus where a midline scalp incision was made. Wound margins were infused with a long-lasting local anesthetic (bupivacaine). The scalp and muscles were reflected, and small craniotomies were drilled in the appropriate locations. After the injection of tracer substances (described below), the scalp was sutured and the animals were allowed to recover from surgery.

Injections of wheat germ agglutinin-horseradish peroxidase and biocytin. Injections of wheat germ agglutinin-horseradish peroxidase (WGA-HRP) were made into the LGN (4 cases) and the pulvinar nucleus ( 3 cases). The WGA-HRP (5\%; Sigma, St. Louis, MO) was dissolved in normal saline. Injections were made by iontophoresis (2-5 $\mu \mathrm{A}$, for $30 \mathrm{~min}$, pulsed $7 \mathrm{sec}$ on/7 sec off) through glass micropipettes $5-20 \mu \mathrm{m}$ in diameter. For the LGN injections, the micropipelte was angled at $30^{\circ}$ from vertical and inserted into the brain. Layers within the LGN were selected for injection by recording through the injection pipette the responses of neurons to flashes of light to the left or right eye. For the pulvinar injections, the micropipette was positioned into the brain using stereotaxic coordinates.

Injections of biocytin were made into layer VI of striate cortex in 13 animals. Biocytin injections were made by iontophoresis $(1.7-2.0 \mu \mathrm{A}$, for 4-6 min, pulsed $7 \mathrm{sec}$ on $/ 7 \mathrm{sec}$ off) through glass micropipettes $8-12 \mu \mathrm{m}$ in diameter. The biocytin (5\%; Sigma) was dissolved in normal saline. Micropipettes were positioned into layer VI according to appropriate depth, and each hemisphere received one to five biocytin injections spaced no closer than $2 \mathrm{~mm}$ apart.

The WGA-HRP- and biocytin-injected animals were killed after a $2 \mathrm{~d}$ survival by administcring an overdose $(0.5 \mathrm{ml})$ of sodium pentobarbital
$(10 \mathrm{mg} / \mathrm{ml}$, i.p.). After cessation of breathing, animals were perfused with saline followed by $4 \%$ paraformaldehyde in $0.1 \mathrm{~m}$ phosphate buffer $(\mathrm{PB})$, $\mathrm{pH}$ 7.4. After fixation, brains were rinsed in a $10 \%$ solution of sucrose in PB and immersed overnight in a $20 \%$ solution of sucrose in PB.

Histology. For the WGA-HRP experiments, brains were sectioned coronally at $50 \mu \mathrm{m}$ on a freezing microtome. Sections were processed for HRP using tetramethylbenzidine as the chromagen (Mesulam, 1977). then mounted onto gel-subbed slides, lightly counterstained with thionin, quickly dehydrated with alcohol, cleared with xylene, and coverslipped in pcrmount.

Biocytin-injected brains were sectioned coronally at $40 \mu \mathrm{m}$ on a freezing microtome, rinsed in PBS, and placed in a solution of $10 \%$ methanol and $1 \% \mathrm{H}_{2} \mathrm{O}_{2}$ for $30 \mathrm{~min}$. Sections then were removed, rinsed in PBS, and transferred to a solution of Triton X-100 [0.75\% in PBS with $1 \%$ normal rabbit serum (NRS) for $2 \mathrm{hr}$. After this step, sections were rinsed and incubated overnight in a solution of goat anti-biotin (Vector Laboratories, Burlingame, $\mathrm{CA} ; 1: 10,000$ in PBS with $1 \%$ NRS and $0.1 \%$ Triton $\mathrm{X}-100$ ). The next day, sections were rinsed and incubated for $2 \mathrm{hr}$ each in solutions of biotinylated rabbit anti-goat (Vector; 1:200 in PBS with $1 \%$ NRS and $0.1 \%$ Triton $\mathrm{X}-100)$, the avidin component (A) of an $\mathrm{ABC}$ kit (Vector; 1:400 in PBS), and the biotin/HRP component (B) of the ABC kit (1:400 in PBS) with rinses between incubations. The biotinylated rabbit anti-goat, $A$ and $B$ solutions, were saved, and the tissue was incubated in each a second time for $1 \mathrm{hr}$. Sections were reacted for peroxidase histochemistry using diaminobenzidine (Sigma; $0.05 \%$ in PBS with $\left.0.17 \% \mathrm{H}_{2} \mathrm{O}_{2}\right)$ containing nickel $(0.02 \%)$ and cohalt $(0.028 \%)$ for intensification (Adams, 1981). Sections then were mounted and allowed to dry on gel-coated slides before being counterstained with thionin, 


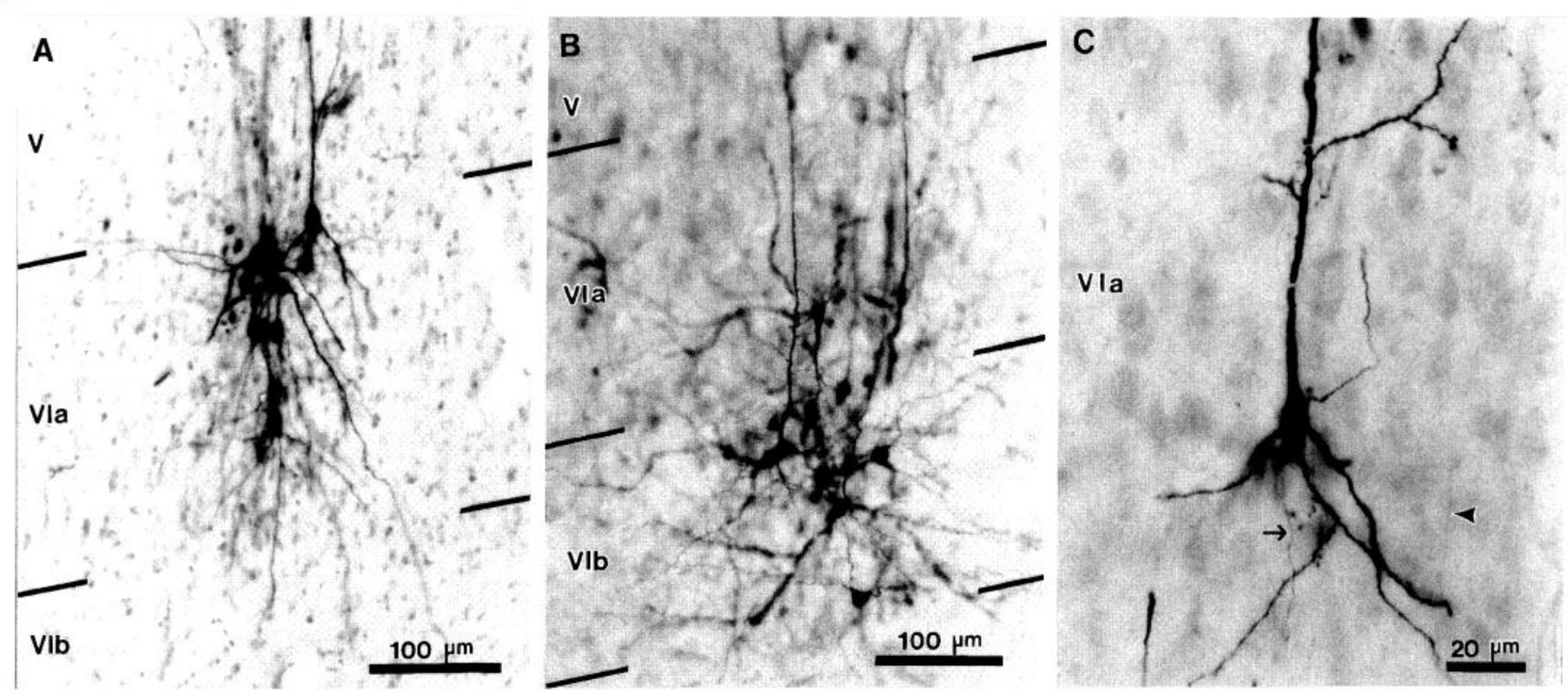

Figure 4. Photomicrographs of striate cortex showing examples of biocytin injection sites restricted either to layer $V I a(A, C)$ or to layer $V I b(B)$. The neurons at the injection sites are filled with reaction product such that the finest details of their processes are revealed. The arrow in $C$ points to the descending axon, and the arrowhead points to a recurrent collateral axon ascending toward layer IV.

\section{A}

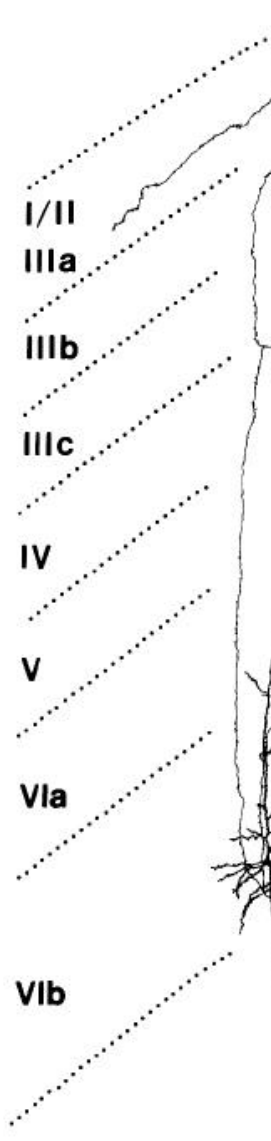

B

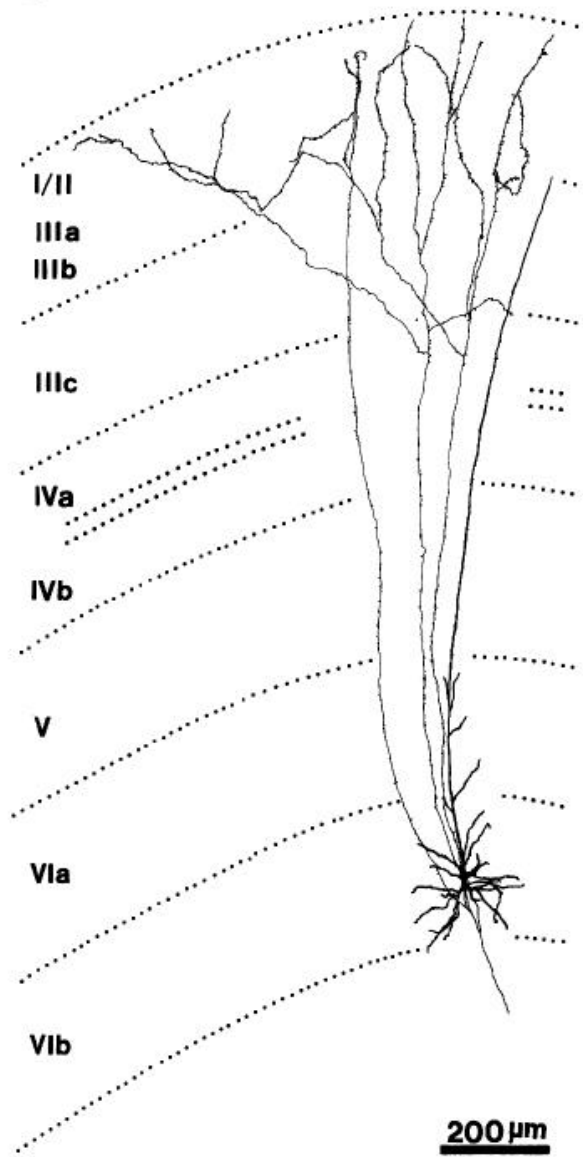

C

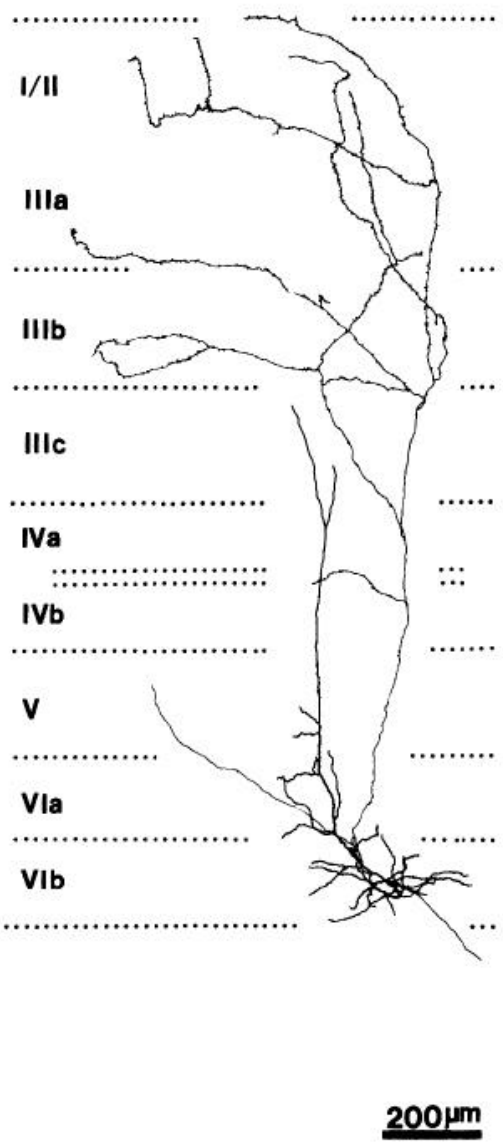

Figure 5. Camera lucida drawings of three individual layer $V I b$ pyramidal neurons $(A-C)$ and their intrinsic projections labeled with biocytin. Each of the neurons has a single axon that exits the soma and enters the white matter. From this axon, one to three collaterals turn and ascend through layers $V I, V$, and $I V$ before branching extensively in the supragranular layers, primarily layers $I I b-I$. The terminal fields of these axons extend for $\sim 500 \mu \mathrm{m}$ parallel to the cortical surface. 
A

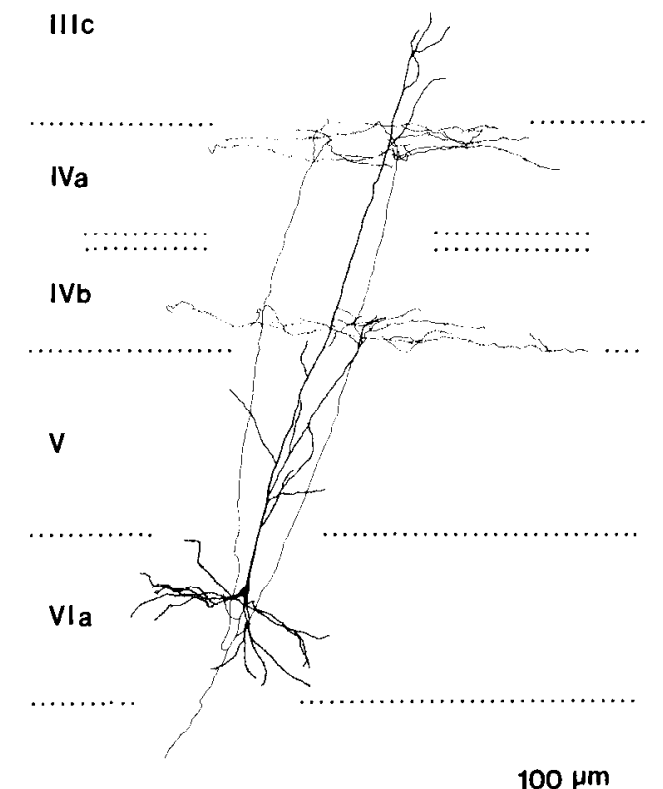

C

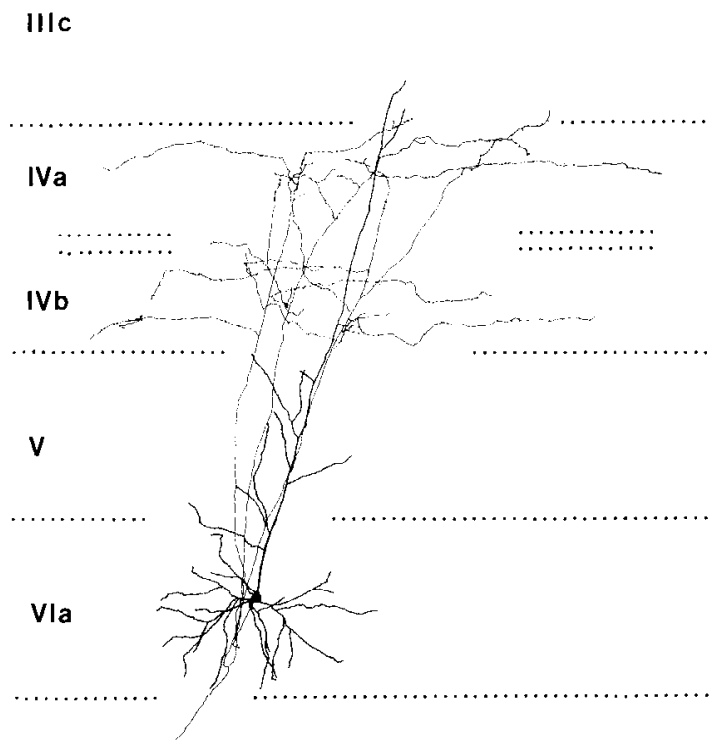

$100 \mu \mathrm{m}$

\section{B}

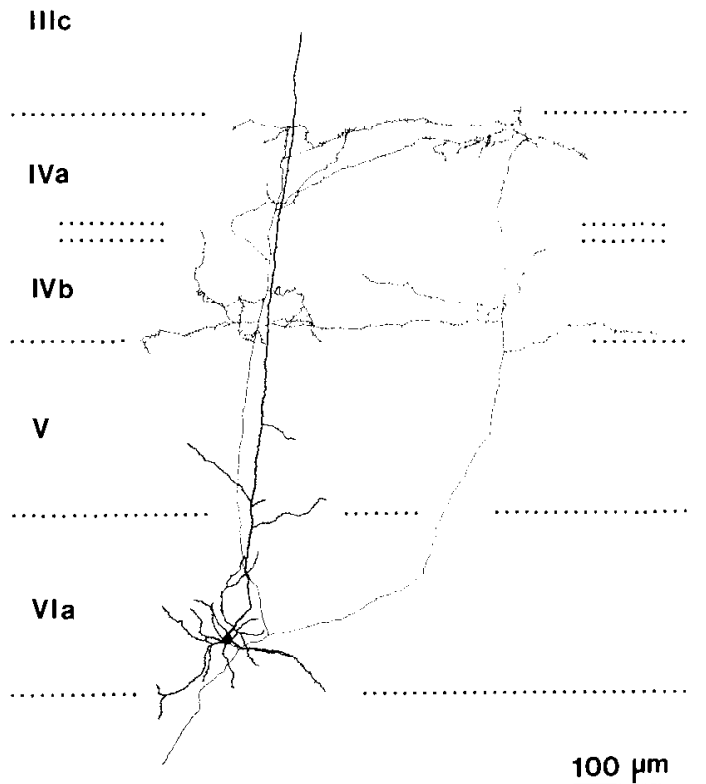

D

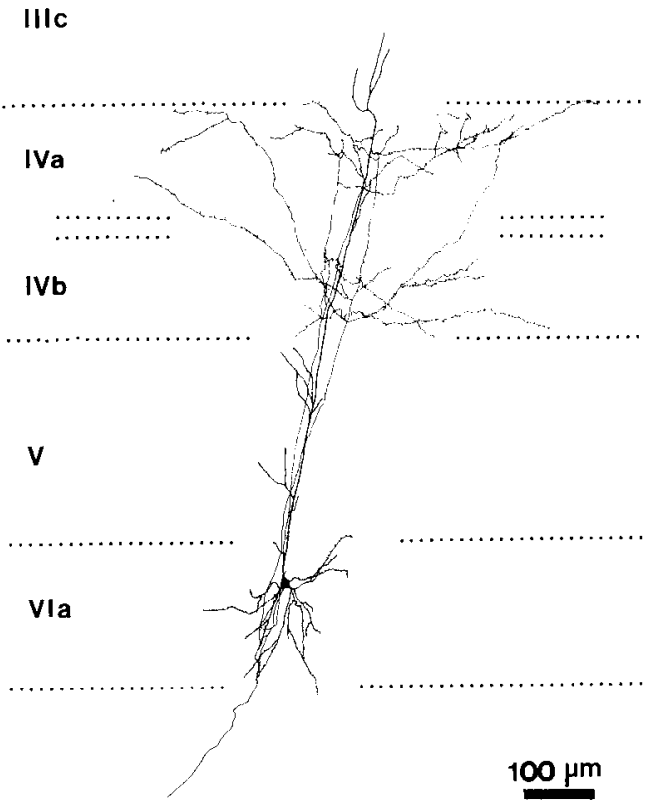

Figure 6. Camera lucida drawings of four biocytin-labeled layer VIa pyramidal neurons with intrinsic projections biased toward the outer edges of layer $I V$. Each of the neurons gives rise to terminals in both $I V a$ and $I V b$. The neurons shown in $A$ and $B$ give rise to axons that are restricted to the outermost edges of $I V a$ and $I \mathrm{Vb}$. The neurons shown in $C$ and $D$ give rise to axons that arborize more widely across $I V a$ and $I V b$. These axons, however, still eschew the inner edges of $I V a$ and $I V b$.

dehydrated with alcohol, cleared with xylene, and coverslipped in permount.

Dafa analysis. The sublaminar distribution of retrogradely labeled neurons in layer VI of striate cortex was evaluated after the injections of WGA-HRP into the LGN and pulvinar nucleus. Injection sites and labeled cells were examined with both bright- and dark-ficld illumination. Drawings of the location of labeled neurons were made from representative sections using a camera lucida and $20 \times$ or $63 \times$ objective. Histo grams were made to quantify the sublaminar distribution of labeled cells across the depth of layer VI. This was accomplished by counting the 
A

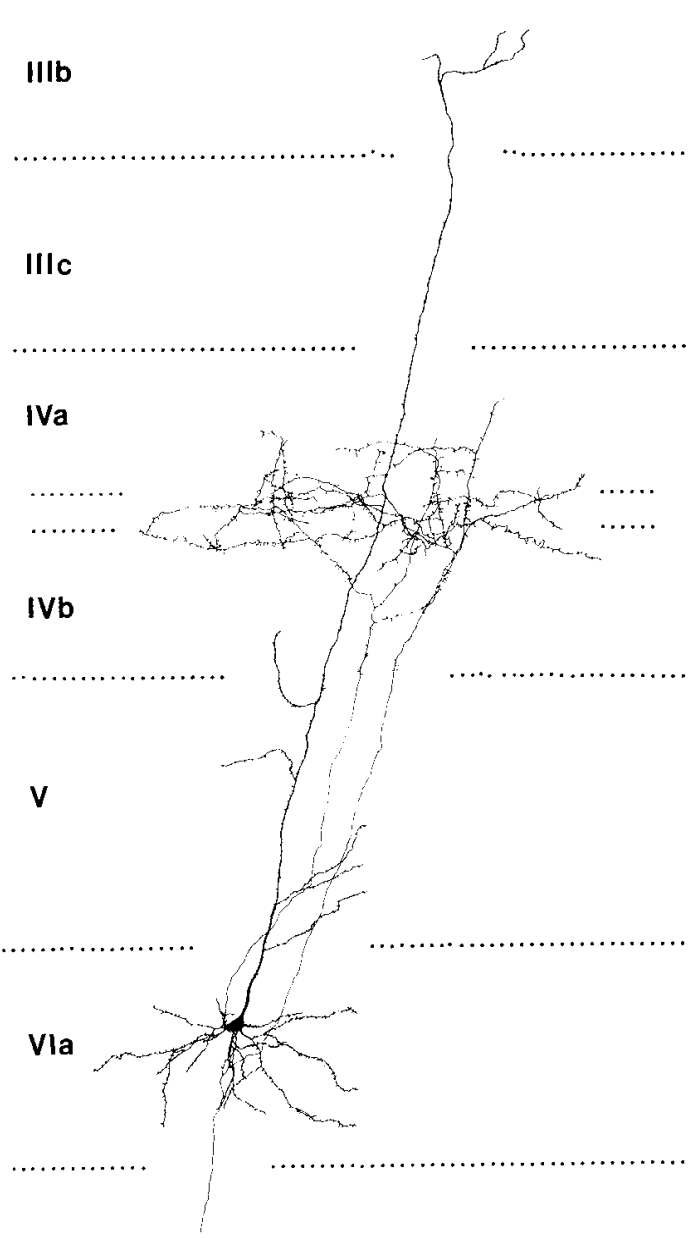

B

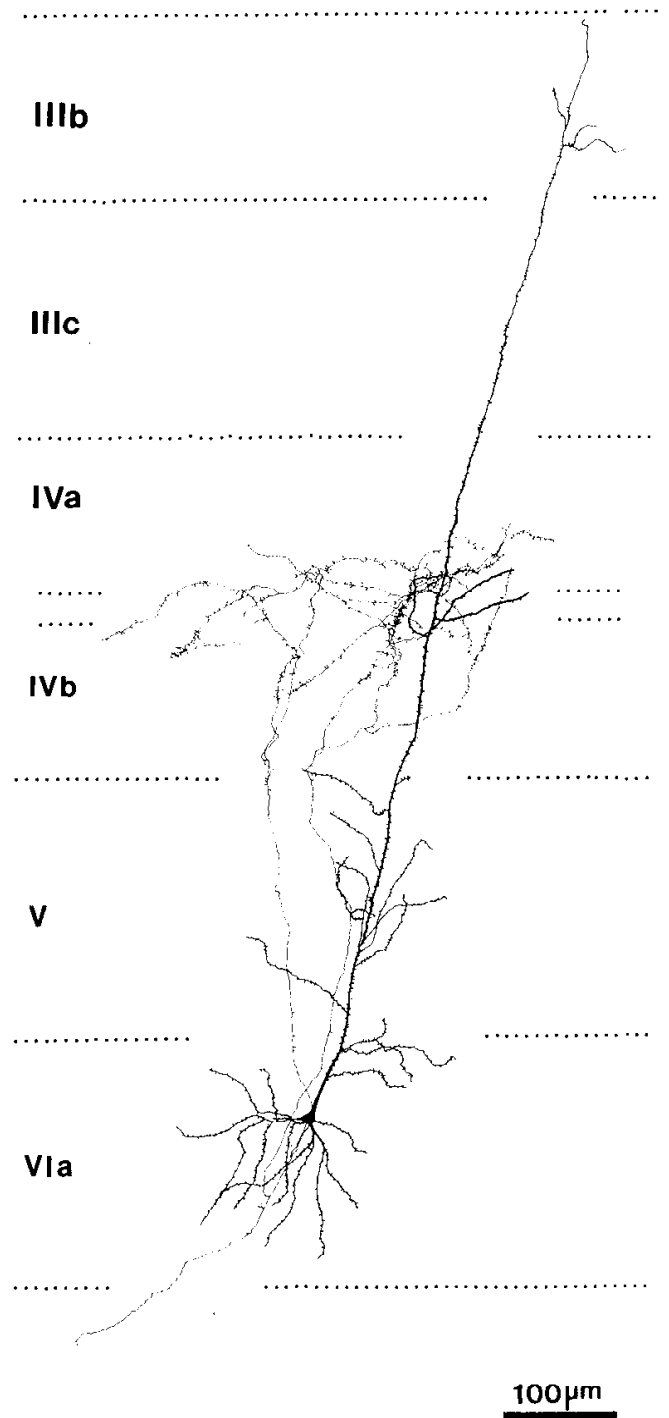

Figure 7. Camera lucida drawings of two biocytin-labeled layer Vla pyramidal neurons $(A, B)$ with intrinsic projections that target the middle of layer IV. The intrinsic axons of these neurons arborize in both lower $I V a$ and upper $I V b$.

number of labeled cells contained in each of 10 equal tangential tiers across the depth of layer VI.

To document the extrinsic and intrinsic projections of individual and small populations of layer VI neurons, drawings of biocytin-labeled layer VI neurons and their axons were made using a camera lucida and $63 \times$ oil objective. For the extrinsic axons, histograms were made to quantify the laminar distribution of boutons across the LGN. This was accomplished by counting the number of biocytin labeled boutons located in each of 30 equal tangential tiers across the depth of LGN and then assigning laminar borders to these histograms. For the layer VI neurons projecting only to layer IV, histograms were made to quantify the sublaminar distribution of terminals from individual layer VI neurons across the depth of layer IV. This was accomplished by counting the number of biocytin-labeled boutons located in each of 10 equal tangential tiers across the depth of layer IV.

\section{RESULTS}

\section{Cytoarchitecture of tree shrew striate cortex}

Figure 1 provides a brief review of the major features of the tree shrew striate cortex that are relevant for understanding the connections of layer VI neurons. The most prominent feature of tree shrew striate cortex, and the principal target of layer VI intrinsic axon arbors, is the darkly stained, cell-rich layer IV (Fig. 1). A cell-sparse cleft divides this layer into two tiers, IVa and IVb, which are the targets of ON- and OFF-center LGN pathways, respectively (Harting et al., 1973; Conley et al., 1984; Raczkowski and Fitzpatrick, 1990). As our results show, layer VI neurons also project to the supragranular layers, the largest part of which is occupied by three subdivisions of layer III: the cell-sparse IIIc, which lies immediately adjacent to layer IV; the cell-rich IIIb, which occupies the middle third of layer III; and IIIa, which is intermediate in cell density and borders on the cell-rich layer II. Layer VI is composed of subdivisions that differ in their cell density: a prominent cell-rich zone (VIa) that lies adjacent to layer V, and a cell-sparse zone (VIb) that extends from the base of VIa into the white matter. The results presented here suggest that neurons in these two subdivisions differ in their pattern of intracortical and thalamic projections.

\section{Organization of layer $\mathrm{VI}$ intracortical projections}

Large injections of biocytin into layers VIa and VIb were made to examine the total pattern of layer VI projections to the overlying cortical layers. An example of such an injection site and the resulting pattern of labeled terminals are shown in Figure $2 \mathcal{A}$. 

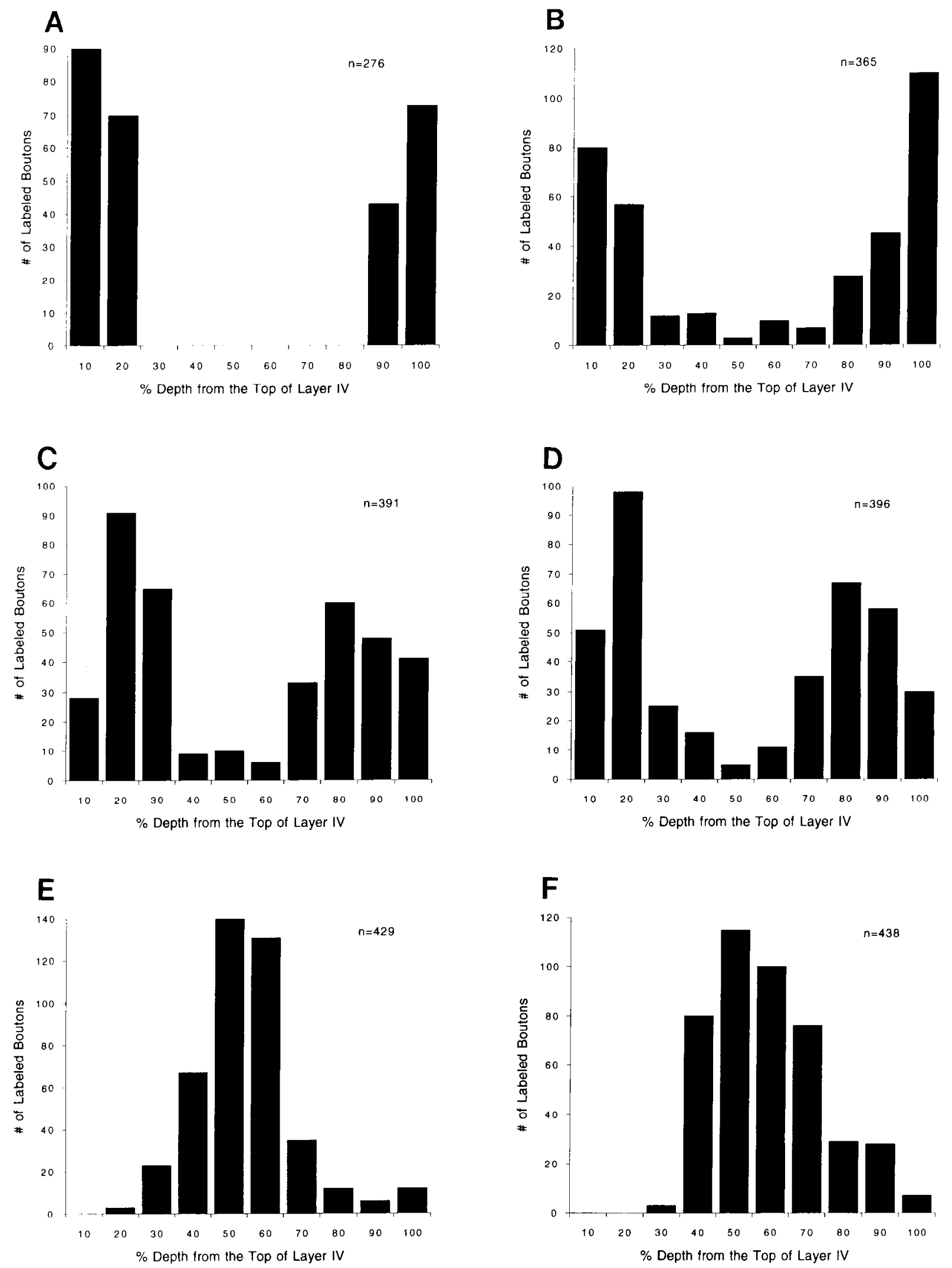

Figure 8. Histograms showing the distribution of labeled boutons across the depth of layer IV for each of the neurons in Figures 6 and $7 . A, B$, Histograms for the neurons shown in Figure 6, $A$ and $B$. These histograms show a clear bias of terminals toward the outermost edges of layer IV with the middle of layer IV containing few, if any, terminals. $C, D$. Histograms for the neurons shown in Figure $6, C$ and $D$. These histograms also demonstrate an absence of terminals in the middle of layer IV; however, terminals are not as restricted to the outermost edges as those shown in $A$ and $B$. $E, F$. Histograms for the neurons shown in Figure 7, $A$ and $B$. These histograms clearly show a peak in the number of terminals present in the middle of layer IV ind few, if any, terminals in the outer edges of layer IV. In each of the above described histograms, the number of terminals in IVa and IVb is approximately equal. 


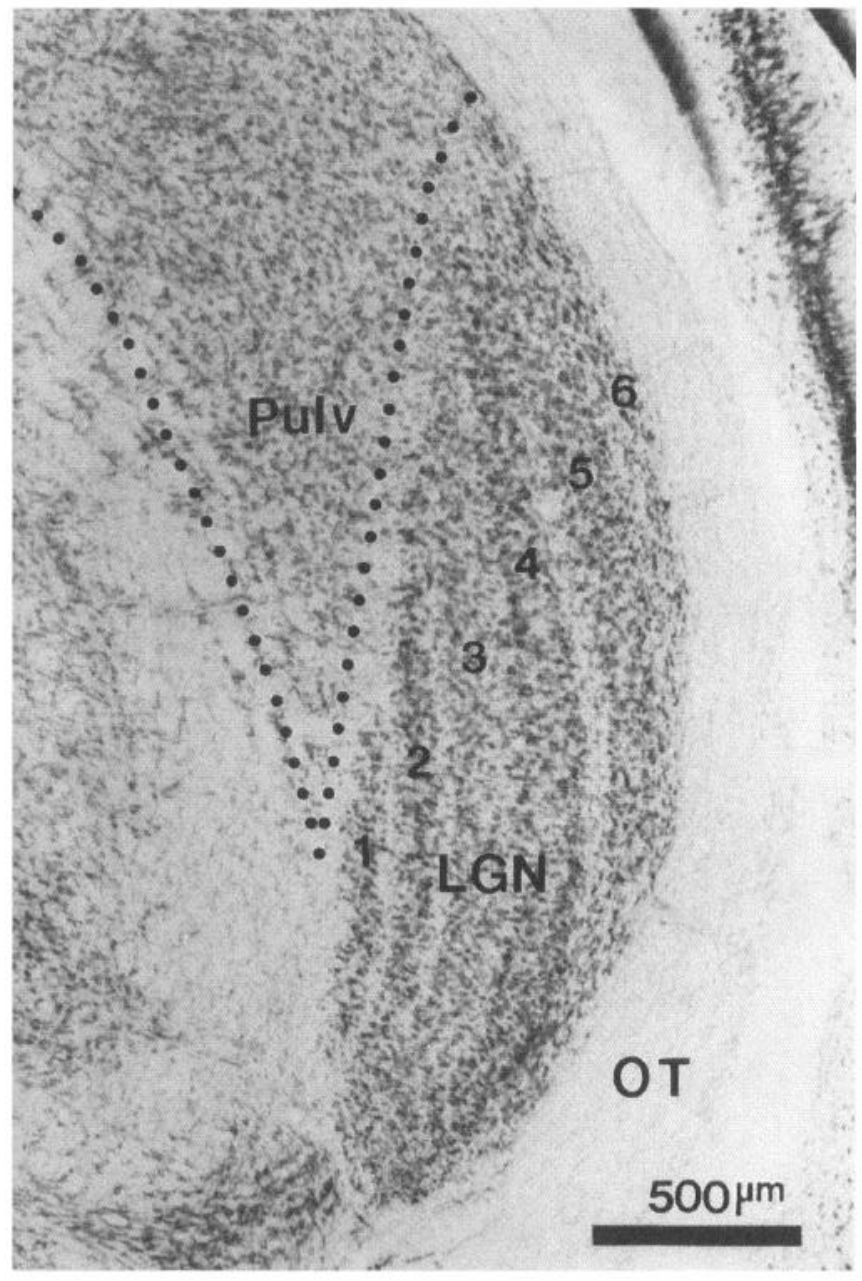

Figure 9. Photomicrograph of a Nissl-stained section of thalamus showing both the $L G N$ and the pulvinar nucleus (Pulv). The LGN contains six well defined layers. Layers $1,2,4$, and 5 project to layer IV of striate cortex. Layers 3 and 6 project to the supragranular layers of striate cortex.

Labeled fibers rise in a radial manner away from the layer VIa/ $\mathrm{VIb}$ injection site and give rise to a dense band of labeled terminals that extends across the depth of layer IV. In addition, a second distinct band of labeled terminals is present in layer IIIb. Both of these labeled bands contain a dense network of finecaliber axons and boutons (Fig. $2 B$ ). Consistent with the descriptions of layer VI axon arbors in other species (Lund and Boothe, 1975; Gilbert and Wiesel, 1979; Katz, 1987; Anderson et al., 1993), most of the boutons are attached to the axonal branches by short, thin stalks (arrow). In addition to the dense bands of labeling in layers IV and IIIb illustrated in Figure $2 A$, a less dense plexus of labeled fibers extends above layer IIIb into layer I. These fibers are similar in morphology to those terminating in the deeper layers.

Smaller injections of biocytin restricted to either VIa or VIb show that the projections to layer IV and layer III arise from different populations of layer VI neurons. A total of 13 injections of biocytin were restricted to layer VIb; in each case, labeled terminals were found primarily in layers IIIb-I. The distribution of axon arbors from a small population of layer VIb neurons is illustrated in Figure $3 B$. The ascending axons of layer VIb neurons pass through layer $\mathrm{V}$ without branching. In layer IV and in layer IIIc, the main axonal trunks begin to branch and their collaterals ascend into layer IIIb to form a dense terminal field. The axons of some layer VIb neurons give rise to axon collaterals with a small number of terminals in layer IV; when present, this minor projection usually is directed to the region surrounding the cleft (lower IVa, upper IVb).

In contrast, injections of biocytin restricted to layer VIa result in a dense plexus of labeled terminals in cortical layer IV. Twenty injections of biocytin were restricted to layer VIa of striate cortex and, in each case, labeled fibers and terminals were present in layers IVa and $\mathrm{IVb}$ with only an occasional labeled fiber extending into the supragranular layers. The distribution of axon arbors from a small population of layer VIa neurons is shown in Figure $3 A$.

To examine the morphology of individual layer VI neurons that contribute to the patterns just described, we reconstructed the dendritic processes and axon arbors of 8 single VIb neurons and 16 VIa neurons from our smallest biocytin injections (Fig. 4). All of the neurons that we reconstructed were pyramids, and the results to be presented are confined to a description of this neuronal class. These neurons had clearly defined apical and basilar dendritic fields, the processes of which were studded with spines. Each of these neurons also had a single axon that descended from the soma and entered the white matter. Before entering the white matter, all of the pyramidal cell axons gave rise to recurrent collaterals (typically $2-3$ ) that turned and ascended through layer VI to terminate in the more superficial cortical layers.

Examples of biocytin-labeled neurons with cell bodies located in layer VIb are shown in Figure 5. In each case, the ascending axonal trunks have a predominantly vertical trajectory until they reach layer IIIb, where they branch repeatedly to form a plexus that extends throughout layers IIIb-I. The pyramidal neurons in layer VIb appear to be homogenous with respect to the distribution of their axon arbors. We found no evidence for subtypes of layer VIb neurons with axons restricted to less than the full width of layers IIIb-I.

All of the reconstructed neurons with cell bodies located in layer VIa gave rise to axon arbors that terminated in both subdivisions of layer IV (IVa and IVb). Thus, individual layer VIa neurons show little selectivity for the ON and OFF pathways within layer IV. However, two distinct classes of layer VIa neurons could be identified based on the sublaminar distribution of their terminals within each subtier of layer IV. One class has terminal fields that are biased toward the outer edges of layer IV (the upper part of IVa and the lower part of IVb); the other has terminal fields that are biased toward the cell-sparse cleft of layer IV (the lower part of IVa and the upper part of IVb).

Examples of layer VIa pyramidal cells with axon terminal fields biased toward the edges of layer IV are shown in Figure 6. In each case, the recurrent axons rise through layer $\mathrm{V}$ and arborize in two separate tiers leaving the region surrounding the cleft relatively free of labeled terminals. The extent to which the terminal arbors of these cells encroached on the middle of IVa and IVb varied from cell to cell. At one extreme are the cells such as that shown in Figure $6, A$ and $B$, the axon terminals of which are virtually confined to the outer third of each tier. At the other extreme are cells such as those shown in Figure 6, $C$ and $D$, the axon terminals of which spread more widely across the depth of each tier and yet still spare the region around the cleft.

In contrast, Figure $7, A$ and $B$, shows examples of layer VIa neurons with projections that are biased toward the middle of layer IV. These neurons give rise to recurrent axons with a single 
A

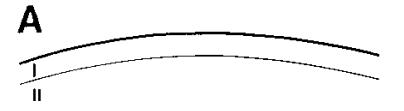

Ha

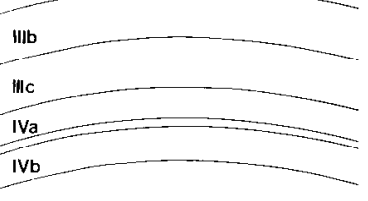

$\mathrm{V}$

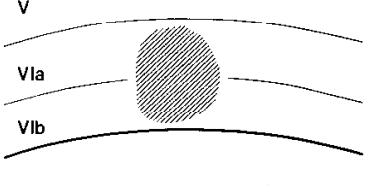

$500 \mu \mathrm{m}$
B

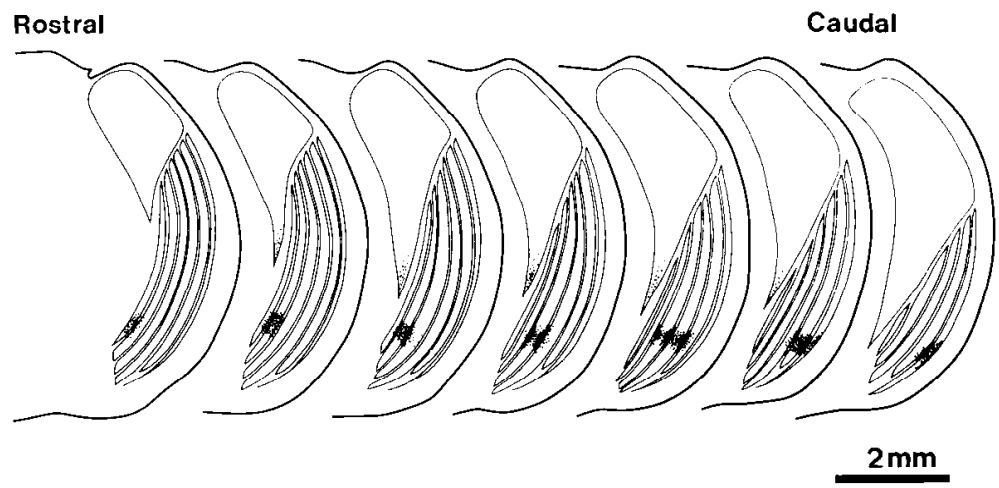

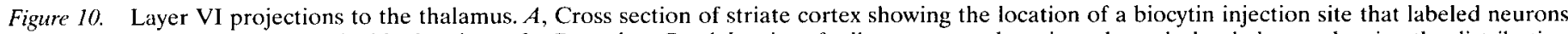

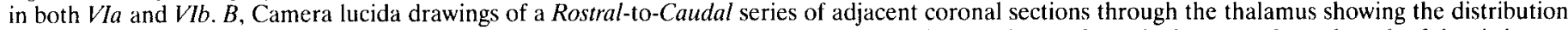

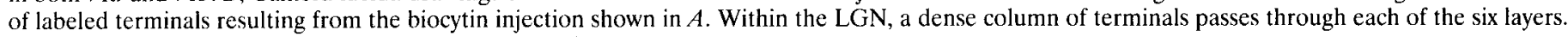
Labeled terminals also are present in the pulvinar nucleus.

terminal field that occupies the cleft and the immediately adjacent parts of IVa and IVb.

The difference between these two classes in the sublaminar arrangement of axon terminal fields is illustrated in quantitative form in Figure 8. The histograms in this figure show the number of labeled boutons as a function of depth from the top of layer IV for each of the axons illustrated in Figures 6 and 7. Those illustrated in Figure 6 have two peaks at the edges of layer IV, whereas those illustrated in Figure 7 have a single peak at the midpoint of the layer.

In addition to differences in the distribution of axon terminals, we also noted more subtle, but consistent differences in the distribution of the apical dendrites of these two classes of VIa neurons. All of the neurons gave rise to apical dendrites that arborized in layer $\mathrm{V}$. However, those with axon arbors in the middle of layer IV gave rise to dendrites that also arborized in the middle of layer IV and in layer IIIb. In contrast, those with axon arborizations at the edges of layer IV had apical dendrites that did not extend above the lower part of layer IIIc and, in some cases, gave rise to a small tuft in this region.

\section{Organization of layer VI projections to the thalamus}

Before describing the pattern of axonal projections to the thalamus, it is necessary to review the organization of the tree shrew LGN. The LGN in the tree shrew is a prominent structure with six well defined layers (Fig. 9). Layers 1, 2, 4, and 5 are the source of projections to layer IV of striate cortex (Harting et al., 1973; Conley et al., 1984; Raczkowski and Fitzpatrick, 1990). Layers 1 and 2 are a matched-pair that provide layer IVa with ON-center information from the ipsilateral and contralateral eyes, respectively (Norton, 1982; Conway and Schiller, 1983; Holdefer and Norton, 1995). Layers 4 and 5, also a matched-pair, provide layer IVb with OFF-center information from the contralateral and ipsilateral eyes, respectively. Layers 3 and 6 are the small-cell intercalated layers that project directly to the supragranular layers of striate cortex (Carey et al., 1979; Conley et al., 1984; Usrey et al., 1992). Layers 3 and 6 both receive input from the contralateral eye and these layers contain neurons with ON/OFF response properties (Norton, 1982; Conway and Schiller, 1983).

As a first step toward understanding the organization of projections from layer VI neurons to the thalamus, we examined injections of biocytin that included both VIa and VIb and reconstructed the total pattern of labeled axon arbors in the thalamus. An example of such an injection and the resulting pattern of labeled terminals is shown in Figure 10. The injection site is centered at the layer $\mathrm{VIa} / \mathrm{VIb}$ border and extends across most of the depth of layer VI. Within the thalamus, the most prominent feature of the layer VI projection is a dense column of labeled terminals that passes through each of the six LGN layers. The column of labeled terminals is oriented at an oblique angle to the coronal plane of section; thus, a rostral-to-caudal series of sections is necessary to reveal the entire projection pattern. Labeled terminals are also present in the interlaminar zones that separate each of the layers. Indeed, consistent with previous reports (Brunso-Bechtold et al., 1983), the projection to the interlaminar zones often appears more robust than the projection to the layers.

A second cluster of labeled axons and terminals lies immediately medial to the LGN in the most ventral portion of the pulvinar nucleus. Previous studies identified this region as the "capsule of the pulvinar nucleus" and demonstrated that it is a source of projections to the supragranular layers of striate cortex (Carey et al., 1979). The labeled axons in the LGN and the pulvinar nucleus are virtually identical in appearance. The terminal swellings are attached to axonal branches primarily by short, thin stalks in a drumstick-like arrangement. Terminals are also present en passant, although they are much less abundant.

Smaller injections of biocytin restricted to either VIa or VIb resulted in different patterns of labeled terminals in the thalamus. Figure 11 shows three examples of the distribution of layer VIb axons in the thalamus. These examples include a small population of layer $\mathrm{VIb}$ axons and two individual layer VIb axons. In each case, layer VIb axons give rise to collaterals with terminal boutons in the pulvinar nucleus (arrowheads) before entering the LGN. Within the LGN, axons extend across all six layers arborizing preferentially in laycrs 3 and 6 and to some extent in the interlaminar zones. The histograms that accompany each of the reconstructions in Figure 11 provide a quantitative assessment of the distribution of labeled terminals across the depth of the LGN. Within layer 3, the number of terminals peaks near the edges of the layer and in the interlaminar zones on each side of the layer. 


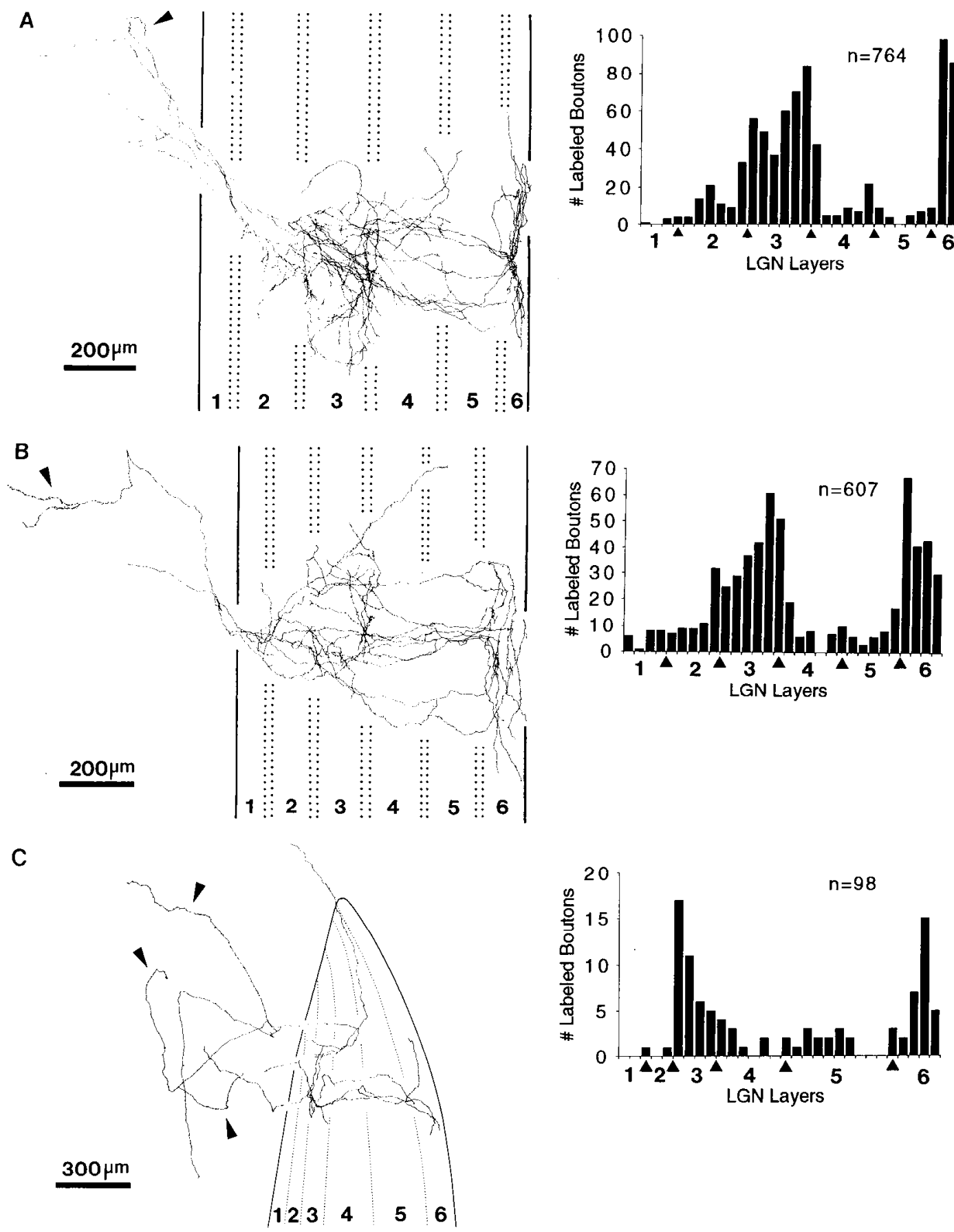

Figure 11. Camera lucida drawings of biocytin-labeled layer VIb axons arborizing within the LGN and the pulvinar nucleus. $A$, Reconstruction of the axonal arbors from a small population of layer VIb neurons. $B, C$, Reconstructions of the axonal arbors from individual layer VIb neurons. In each reconstruction, axons enter the LGN and arborize preferentially in layers 3 and 6 . The histograms show the distribution of labeled boutons across the depth of the LGN for each reconstruction. In each case, the number of labeled boutons peaks within layers 3 and 6 . Before entering the LGN, each of the axons also gives rise to terminals (arrowheads) within the pulvinar nucleus.

In contrast, axons labeled after injections into layer VIa pass through the pulvinar nucleus without arborizing and give rise to a different pattern of arborization in the LGN. As shown in Figure 12 , after injections in layer VIa, the density of labeled terminals is greatest in layers 1,2,4, and 5 and in the interlaminar zones; only a few labeled terminals are present in layers 3 and 6 . In general, the OFF layers (layers 4 and 5) contain more labeled terminals than the ON layers (layers 1 and 2), but individual axons always terminated in both the ON and OFF layers. Although our sample size is small, individual axons appeared to show an ocular bias in their terminal fields: some axons terminated more heavily in the ipsilateral set of layers (layers 1 and 5), whereas others terminated more heavily in the contralateral set of layers (layers 2 and 4).

These results suggest that neurons in layer VIa and VIb differ in their pattern of projection to the LGN. Neurons in layer VIa terminate in the principal layers of the LGN, whereas those in 

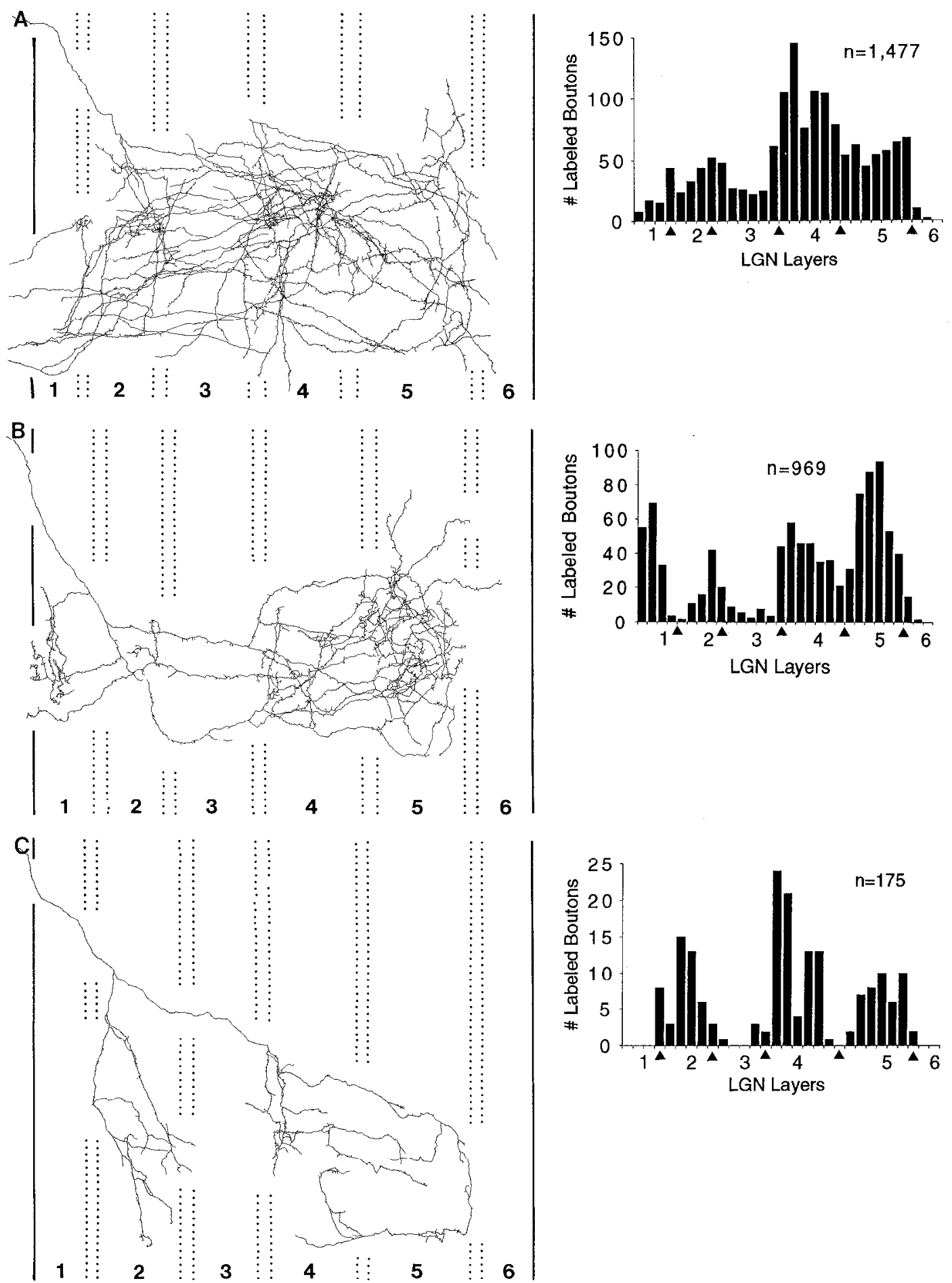

Figure 12. Camera lucida drawings of biocytin-labeled layer VIa axons arborizing within the LGN. A, Reconstruction of the axonal arbors from a small population of layer VIa neurons. $B, C$, Reconstructions of the axonal arbors from individual layer VIa neurons. Each of the axons in these reconstructions avoids arborizing in LGN layers 3 and 6 . Instead, the axons arborize to varying extents in layers $1,2,4$, and 5 . The histograms show the distribution of labeled boutons across the depth of the LGN for each reconstruction. Bouton counts are greatest outside layers 3 and 6 .

layer VIb terminate primarily in layers 3 and 6 . In addition, layer VIb neurons send collaterals to the pulvinar nucleus.

In a final set of experiments, we attempted to confirm these results by using retrograde tracers to examine the distribution of labeled cells in layer VI after injections into the thalamus. Limiting injections of retrograde tracers to LGN layers 3 and 6 proved to be too difficult, therefore, we decided to try a simpler experi- ment and compare the distribution of ncurons projecting to the pulvinar nucleus with those projecting to the LGN. Because only the layer VI axons that terminate in LGN layers 3 and 6 had collaterals that terminated in the pulvinar nucleus, we expected to find pulvinar-projecting neurons limited to VIb and LGNprojecting neurons distributed throughout the depth of layer VI. As illustrated in Figure 13, after injections of retrograde tracers 
Figure 13. Photomicrographs showing examples of WGA-HRP injection sites in the LGN and the pulvinar nucleus (Pulv) and the resulting pattern of labeled neurons in layer VI of striate cortex. $A$, An example of an injection of WGA-HRP restricted to the $L G N$. To better illustrate the LGN layers, an injection of WGA-HRP also was made into the contralateral eye. $B$, Neurons in layer VI of striate cortex labeled by the LGN injection shown in $A$. Labeled neurons are present across the depth of layer VI with the density greatest in layer $V I a$. $C$, An example of an injection of WGA-HRP made into the pulvinar nucleus (Pulv) without involvement of the $L G N$. D, Neurons labeled in layer VI of striate cortex by the pulvinar injection shown in $C$. Labeled neurons are located almost exclusively in layer $V I b$.
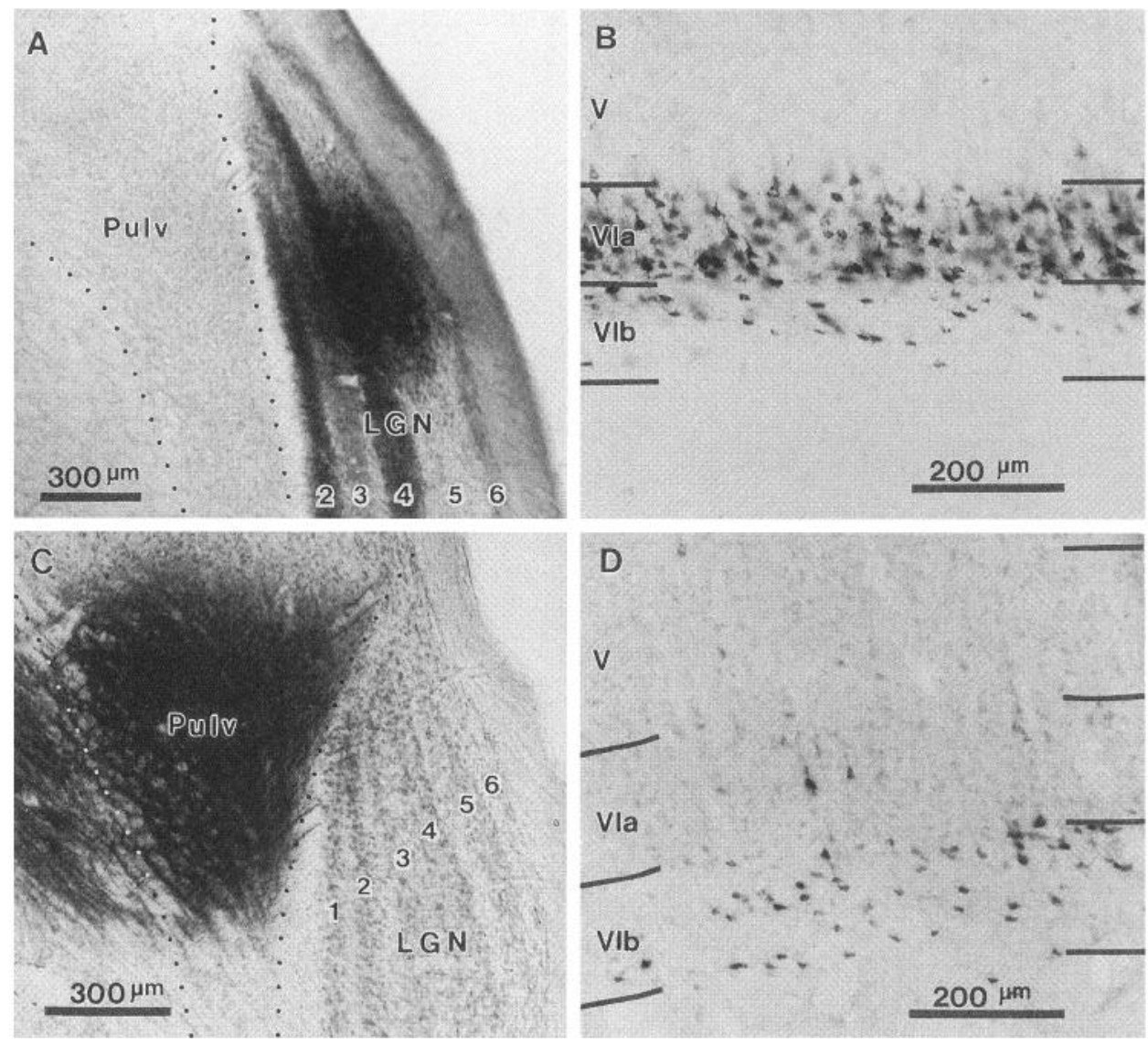

(cholera toxin or WGA-HRP) into the LGN, labeled neurons were found throughout layers VIa and VIb. In contrast, after injections into the pulvinar nucleus, labeled neurons were restricted primarily to layer VIb (Fig. 13). In addition to the labeled cells in layer VIb, injections into the pulvinar nucleus also labeled a small population of neurons in layer $\mathrm{V}$ (not shown).

\section{DISCUSSION}

These results show that layer VI of tree shrew striate cortex consists of two distinct sublaminae that differ in the distribution of their intracortical and thalamic projections (Fig. 14). Neurons in layer VIa terminate in cortical layer IV, whereas neurons in layer VIb terminate throughout the supragranular layers, layers I-III. The selectivity of layer VI subdivisions for the granular and supragranular layers also is manifest in their descending projections to the thalamus. Although both tiers contain neurons that project to the LGN, neurons in layer VIa terminate preferentially in the LGN layers that supply layer IV (LGN layers 1, 2, 4, and 5), whereas those in layer VIb terminate in the LGN layers that supply layers I-III (LGN layers 3 and 6). Furthermore, neurons in layer VIb send collaterals to the pulvinar nucleus, which also projects to the supragranular layers.

In contrast to the selectivity shown for the granular and supragranular layers, the axons of layer VI neurons show little selectivity for the ON- and OFF-center subtypes of LGN neurons, either in the LGN or in cortical layer IV. Although individual layer VIa neurons project to both the ON and OFF tiers of layer IV, their terminal fields are limited to narrow strata within each tier, suggesting a selectivity related to other features that characterize the responses of layer IV neurons. In the following sections, we consider the significance of these findings for understanding the role of layer VI neurons in visual processing.

\section{Cortical layer IV and the supragranular layers are targets of separate layer VI pathways}

Because layer IV generally is regarded as the target of layer VI pyramidal cell axons (Lund and Boothe, 1975; Gilbert and Wiesel, 1979; Katz, 1987), the finding that injections of biocytin into layer VI resulted in a dense plexus of terminals in the supragranular layers was a surprise. A modest projection from layer VI to the supragranular layers has been noted in rat visual cortex and, similar to our findings in the tree shrew, this projection appears to originate from the deepest part of layer VI (Burkhalter, 1989). Although a projection from layer VI to the supragranular layers has not been described in carnivores or primates (however, see Wiser and Callaway, 1994), it seems unlikely that such a pathway is unique to tree shrews and rodents. Layer VI in most species consists of subtiers that differ in cell density and Nissl-staining intensity in a manner similar to that in the shrew (Sugita, 1917; O'Leary, 1941; Krieg, 1946; Billings-Gagliardi et al., 1974). Perhaps the proximity of the supragranular-projecting neurons to the white matter and the fact that they are overshadowed by the greater number of cells in layer VIa account for their omission from the descriptions of layer VI projections in other species.

In addition to their location within layer VI, the supragranularand granular-projecting neurons in layer VI also differ in their dendritic morphology and in their descending projections to the thalamus. The latter conclusion is based on evidence that neurons in layer VIa and VIb differ in their thalamic projections (see below) and the observation that all of the local axon arbors of 
A

I
IIIa
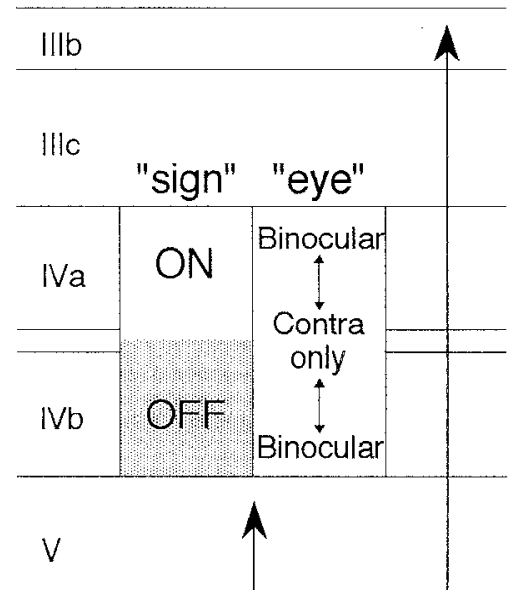

B
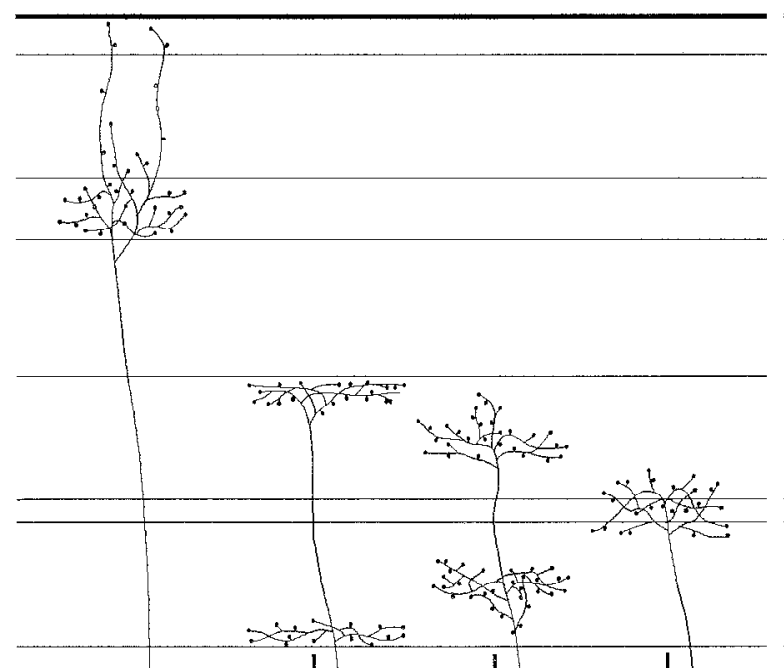

C

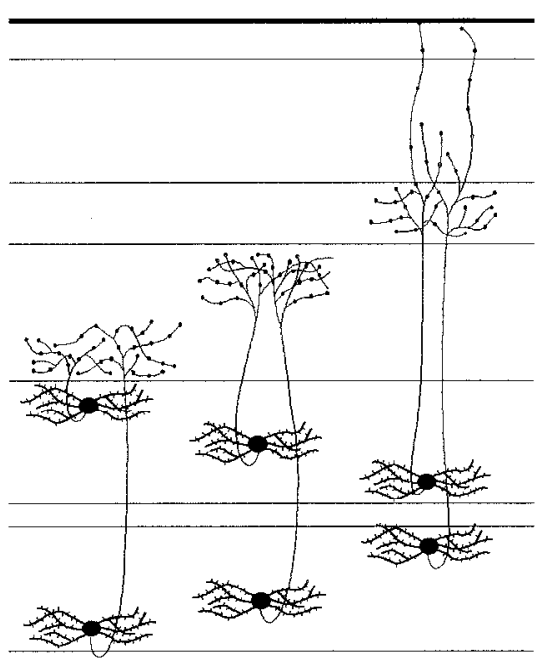

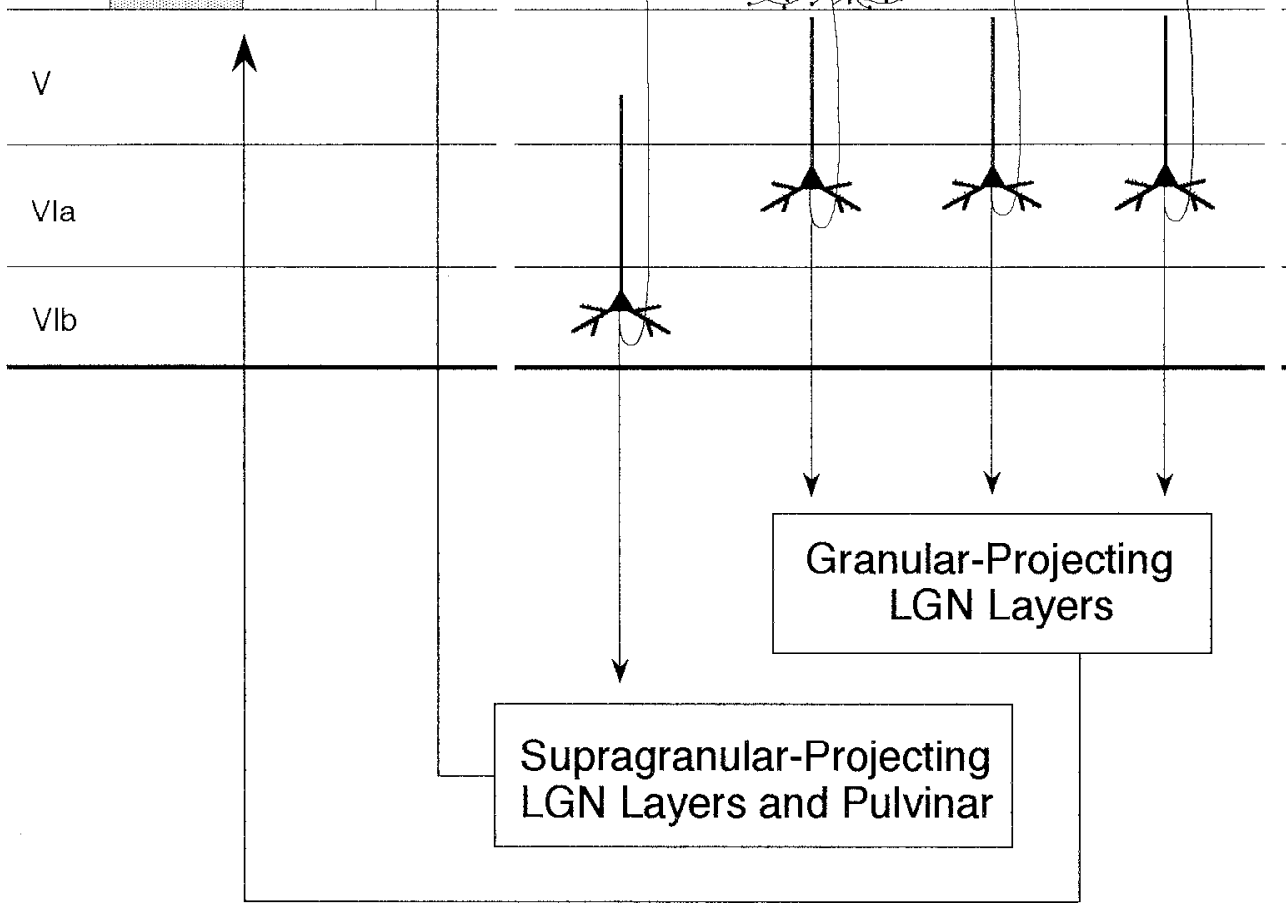

Figure 14. Summary diagram showing the organization of layer VI intracortical and thalamic projections in relation to geniculocortical and layer IV-III pathways. $A$, Different classes of LGN relay cells located in different LGN layers give rise to axons that terminate either in layer IV or in the supragranular layers. Within the LGN to layer IV projection, $O N$ and $O F F$ pathways terminate in $I V a$ and $I V b$, respectively. Layer IV axons are segregated further based on ocularity: axons driven by the contralateral eye terminate throughout the depth of IVa or IVb, whereas axons driven by the ipsilateral eye terminate along the outer edges of IVa or IVb. As a result, neurons located in the outer halves of IVa and IVb are binocular, whereas those located in the inner halves of IVa and IVb are driven by the contralateral eye only. $B$, The results presented show that layer VI consists of two distinct sublaminae that are distinguished by their intracortical and thalamic projections. Neurons in layer VIa give rise to intracortical axons that terminate in layer IV and thalamic axons that terminate in the LGN layers that supply layer IV. In contrast, neurons in laver VIb give rise to intracortical axons that terminate in the supragranular layers and thalamic axons that terminate in the LGN layers that project directly to the supragranular layers. Layer VIb neurons aiso give rise to a collateral projection that terminates within the pulvinar nucleus. $B, C$, Layer VIa neurons arborize in both IVa and IVb; however, within IVa and $\mathrm{IVb}$, these projections are selective for either the inner or the outer edges of the layers. Neurons located in these mirror-symmetric tiers of layer IV (C) terminate in an overlapping and sublaminar-specific manner in the supragranular layers. Layer VIa axons, therefore, appear to be selective for these parallel, layer IV-III pathways.

layer VI neurons in the tree shrew originate as collaterals from axons that enter the white matter. Because we have not traced the entire axon arbor of individual layer VI neurons (i.e., both local and distant terminal fields), we cannot rule out the possibility that some of the layer VI neurons, the local axon arbors of which we reconstructed, project to targets other than the thalamus. How- ever, the most likely nonthalamic target is the claustrum-a structure that receives inputs from $<5 \%$ of the neurons in layer VI of striate cortex, all of which are located in layer VIa (Carey et al., 1980; LeVay and Sherk, 1981; Katz, 1987). Given that LGNprojecting neurons constitute $-50 \%$ of the neurons in layer VIa (Gilbert and Kelly, 1975; Katz, 1987), it seems reasonable to 
conclude that a majority of the neurons in our sample of layer VIa neurons project to the thalamus, and that these neurons have collaterals that terminate in cortical layer IV.

\section{Descending projections of layer VI are selective for the granular- and supragranular-projecting layers of the LGN}

Studies in other species also have provided evidence that the corticogeniculate pathway is composed of subtypes of layer VI cells that differ in their patterns of termination within the LGN (Lund et al., 1975; Conley and Raczkowski, 1990; Fitzpatrick et al., 1994; Murphy and Sillito, 1994). However, these descriptions have focused on the more prominent cell layers in the LGN, those that send their axons to cortical layer IV. The results of this study suggest that the small-cell layers of the LGN, the layers that are the source of projections to the superficial cortical layers, are the targets of a separate descending pathway that arises from neurons in layer VIb. Whether there is a distinct descending pathway to the supragranular projecting layers of the LGN in other species remains unknown. In primates, the small-cell intercalated layers of the LGN, as with the magno- and parvocellular layers, receive a significant contribution from layer VI cells in striate cortex (Lin and Kaas, 1977; Symonds and Kaas, 1978; Wilson and Hendrickson, 1981); however, their scattered location in the interlaminar spaces has made it difficult to determine the source of their layer VI input (Fitzpatrick et al., 1994).

Although the descending projections of neurons in layers VIa and VIb are distinct, it must be acknowledged that the terminal distributions of these two layers overlap within the interlaminar zones of LGN. The interlaminar zones of the tree shrew LGN (as with those in other species) contain the distal dendrites of the relay cells and interneurons, the cell bodies of which lie in the adjacent layers (Brauer et al., 1981; Conley and Wilson, 1992). Thus, the overlap of the two distributions in these regions could signify that some LGN neurons receive synaptic inputs from neurons in both VIa and VIb. Alternatively, the synaptic contacts in the interlaminar zones could be just as specific as the synaptic contacts in the layers; i.e., the terminal fields of layer VI neurons might be selective for the dendritic processes of supragranular- or granular-projecting LGN neurons in the layers as well as in the interlaminar zones. Although this issue cannot be resolved with light microscopic analysis, the pattern of projections to the interlaminar zones favors the latter interpretation. For example, after injections in layer VIb, labeled terminals are numerous in the interlaminar zones that surround LGN layer 3 and sparse in the interlaminar zones between layers 4 and 5 and between layers 1 and 2 . If the distal dendrites of layer 2 and layer 4 neurons receive synaptic contacts from layer VIb neurons, then one would expect to see terminals in all of the interlaminar zones, not just those that surround layer 3 .

In addition to the difference in their laminar distribution within the LGN, neurons in layer VIb are distinguished by the fact that they project to both the LGN and the pulvinar nucleus. A recent study in the rat visual cortex also has demonstrated that individual neurons in layer VIb send collaterals to both the LGN and the pulvinar nuclcus (Bourassa and Deschenes, 1995). Projections to the pulvinar nucleus from the deepest part of layer VI have also been described in the galago (Conley and Raczkowski, 1990). Although they reside in different nuclei, neurons in the pulvinar nucleus and LGN layers 3 and 6 are similar in several respects. Both sets of neurons receive terminals from the superficial layers of the superior colliculus (Harting et al., 1973; Fitzpatrick et al.,
1980), and they are both sources of projections that terminate in the supragranular layers of striate cortex (Carey et al., 1979; Conley et al., 1984; Usrey et al., 1992). Thus, the correspondence between the local connections of layer VI neurons and the distribution of their terminals in the LGN may reflect a more general rule that underlies the connections of corticothalamic neurons.

\section{Relation of layer VI projections to the sublaminar organization of tree shrew layer IV}

Our results also show that there are subclasses of layer VIa neurons that differ in the distribution of their axon terminals within layer IV. Unlike LGN afferents that restrict their terminal fields to either IVa or IVb, individual layer VIa neurons project to both IVa and IVb, terminating in either the outer or the inner parts of each sublayer. The strata of layers IVa and IVb identified by the terminal fields of different classes of layer VI neurons are similar to strata that have been identified in studies of the organization of projections from layer IV to the supragranular layers (Muly and Fitzpatrick, 1992) (Fig. 14). Neurons with cell bodies that lie at the outer edges of layer IV (upper IVa and lower IVb) have axon terminal fields that are restricted to the deepest part of layer IIIc; in contrast, neurons with cell bodies that lie in the inner part of each sublayer (lower IVa, upper IVb) give rise to axons that ascend through IIIc to terminate in layers I-IIIb. A third pathway connects neurons with cell bodies located in the middle of layers IVa and IVb with the upper part of layer IIIc. The similarity in the two patterns suggests that each of the parallel pathways that links layer IV with layers I-III is associated with a distinct class of layer VI neurons.

One feature that distinguishes the response properties of the neurons in these parallel layer IV-III pathways is ocular dominance. Unlike other species in which LGN axons driven by the left and right eye terminate in alternating columns, in the tree shrew, LGN axons driven by the two eyes terminate in a partially stratified manner within IVa and IVb (Fig. 14) (Casagrande and Harting, 1975; Hubel, 1975; Conley et al., 1984; Raczkowski and Fitzpatrick, 1990). Lateral geniculate neurons driven by the contralateral eye terminate throughout the depth of IVa and IVb, whereas LGN neurons driven by the ipsilateral eye terminate along the edges of layer IV. As a result, neurons near the edges of layer IV are binocular, whereas those near the center are driven almost exclusively by the contralateral eye (Kretz et al., 1986). This stratified organization for ocular dominance is recapitulated across the layer IV target zones in the superficial cortical layers: the influence of the ipsilateral eye is greatest in layer IIIc; in contrast, neurons in layers II and IIIa are driven almost exclusively by the contralateral eye (Muly, 1992).

These results suggest that the sublaminar specificity in the innervation of layer IV by layer VIa neurons is related, at least in part, to the property of ocular dominance. Layer VI neurons innervate select populations of $\mathrm{ON}$ and $\mathrm{OFF}$ neurons that have matching ocular preferences. Not only are the terminal fields of layer VIa neurons selective for parallel layer IV-III pathways, their dendritic processes may sample selectively from them as well. Layer VIa neurons with terminal fields in the center of layer IV have apical dendrites that branch in the same region. In addition, some of these dendrites ascend to layer IIIb, where they give rise to a second tuft of branches. In contrast, those with terminal fields near the edges of layer IV rarely have apical dendrites that ascend beyond the lower half of layer IIIc, and many of these have small tufts in this region. The selectivity in dendritic sampling suggests that layer VI neurons have ocular 
preferences that match their target regions. If so, then despite the obvious differences between the laminar arrangement of ocular dominance in shrews and the columnar arrangement in other species, there is an underlying functional similarity. In both cases, differences in ocular preference are established by the pattern of LGN axon terminations and preserved by specificity in the patterns of intracortical circuits (Hubel and Wiesel, 1968, 1977).

Recent studies in the cat suggest that the distribution of layer VI axon arbors in the LGN also is correlated with ocular preference (Murphy and Sillito, 1994). Layer VI neurons driven more strongly by the contralateral eye terminate preferentially in lamina $A$, which receives its input from the contralateral eye, whereas layer VI neurons driven more strongly by the ipsilateral eye terminate preferentially in lamina AI, which receives its input from the ipsilateral eye. Studies in primates have also suggested a correspondence between the ocular preference of layer VI neurons and the distribution of their axon arbors in the LGN (Hendrickson et al., 1978). Although our sample size is small, we also noted that layer VIa axons terminated preferentially in the ipsilateral or contralateral sets of the ON and OFF LGN layers. Perhaps the selectivity of layer VIa neurons for subdivisions of layer IV that differ in their ocular preference is associated with a corresponding selectivity in the pattern of their descending axons.

\section{Functional significance of parallel layer VI pathways}

The results of this study demonstrate striking similarities in the pattern of connections established by the local and descending axon arbors of layer VI neurons. Layer VIa neurons project to layer IV and to the LGN layers that supply layer IV, whereas those in layer VIb project to the supragranular layers and to the LGN neurons that project to these layers. Furthermore, the axons of individual layer VIa neurons terminate in both the ON and OFF subtiers of layer IV and the ON and OFF layers of the LGN, and they exhibit a sublaminar arrangement in both targets that appears related to ocular dominance. 'The correspondence between the arrangement of descending and local connections suggests that layer VI neurons serve some role in coordinating the activity of specific cortical circuits with their sources of thalamic inputs.

What this role might be is not clear, but recent studies of the corticogeniculate pathway by Sillito and colleagues offer an interesting possibility (Sillito et al:, 1994). Although layer VI inputs often may be subthreshold, they can modulate the responsiveness of LGN neurons and influence the timing of the response of a relay cell to the presentation of visual stimuli. For example, the responses of the population of LGN neurons activated by an oriented edge are synchronized, and this synchrony appears to depend on descending projections from visual cortex (Sillito et al., 1994). As a result of the temporal coherence supplied by layer VI neurons, the EPSPs evoked in cortical neurons by this population of LGN neurons might summate, thereby indirectly facilitating the transfer of activity from specific sets of LGN neurons to their cortical targets.

Perhaps the local connections of layer VI neurons serve a similar role in regulating the timing of cortical neuron responses to visual stimuli. Indeed, the fact that the same layer VI ncurons are the source of the corticothalamic and intracortical pathways suggests a tight functional linkage between the two systems in the temporal domain. By coordinating the timing of the responses of distinct populations of cortical neurons and the thalamic neurons that supply them, layer VI neurons could exert a powerful influence on the flow of activity in functionally distinct cortical circuits.

\section{REFERENCES}

Adams JC (1981) Heavy metal intensification of DAB-based reaction product. J Histochem Cytochem 29:775-779.

Anderson JC, Martin KAC, Whitteridge D (1993) Form, function and intracortical projections of neurons in the striate cortex of the monkey Macacus nemestrinus. Cereb Cortex 3:412-420.

Billings-Gagliardi S, Chan-Palay V, Palay SL (1974) A review of laminarion in area 17 of the visual cortex of Macaca mulatta. J Neurocytol $3: 619-629$.

Blasdel G, Lund JS (1983) Termination of afferent axons in macaque striate cortex. J Neurosci 3:1389-1413.

Bourassa J, Deschenes M (1995) Corticothalamic projections from the primary visual cortex in rats: a single fiber study using biocytin as an anterograde tracer. Neuroscience 66:253-263.

Brauer K, Werner L, Winkelmann E, Luth HJ (1981) The dorsal lateral geniculate nucleus of Tupaia glis: a Golgi, Nissl and acetylcholinesterase study. J Hirnforsch 22:59-74.

Brunso-Bechtold JK, Florence SL, Casagrande VA (1983) The role of retinogeniculate afferents in the development of connections between visual cortex and the dorsal lateral geniculate nucleus. Brain Res 312:33-39.

Burkhalter A (1989) Intrinsic connections of rat primary visual cortex: laminar organization of axonal projections. J Comp Neurol 279:171-186.

Carey RG, Bear M, Diamond IT (1980) The laminar organization of the reciprocal projections between the claustrum and striate cortex in the tree shrew, 1upaia glis. Brain Res 184:193-198.

Carey RG, Fitzpatrick D, Diamond IT (1979) Thalamic projections to layer I of striate cortex shown by retrograde transport of horseradish peroxidase. Science 203:556-559.

Casagrande VA, Harting JK (1975) Transneuronal transport of tritiated fucose and proline in the visual pathways of tree shrew Tupaia glis. Brain Res 96:367-372.

Cleland BG, Dubin MW, Levick WR (1971) Sustained and transient neurones in the cat's retina and lateral geniculate nucleus. J Physiol (Lond) 217:473-496.

Conley M, Raczkowski D (1990) Sublaminar organization within layer VI of the striate cortex in Galago. J Comp Neurol 302:425-436.

Conley M, Wilson KF (1992) Dendritic organization of class II (inter) neurons in the dorsal lateral geniculate nucleus of the tree shrew: observations based on Golgi, immunocytochemical, and biocytin methods. J Comp Neurol 319:51-65.

Conley M, Fitzpatrick D, Diamond IT (1984) The laminar organization of the lateral geniculate body and the striate cortex in the tree shrew (Tupaia glis). J Neurosci 4:171-197.

Conway JL, Schiller PH (1983) Laminar organization of the tree shrew lateral geniculate nucleus. J Neurophysiol 50:1330-1342.

Dreher B, Fukada Y, Rodieck RW (1976) Identification, classification and anatomical scgregation of cells with $\mathrm{X}$-likc and $\mathrm{Y}$-like properties in the lateral geniculate nucleus of old-world primates. J Physiol (Lond) 258:433-452.

Fitzpatrick D, Carey RG, Diamond IT (1980) The projection of the superior colliculus upon the lateral geniculate body in Tupaia glis and Galago senegalensis. Brain Res 194:494-499.

Fitzpatrick D, Itoh K, Diamond IT (1983) The laminar organization of the lateral geniculate body and the striate cortex in the squirrel monkey (Saimini sciureus). J Neurosci 3:673-702.

Fitzpatrick D, Lund JS, Blasdel GG (1985) Intrinsic connections of macaque striate cortex: afferent and efferent connections of lamina $4 \mathrm{C}$. J Neurosci 5:3329-3349.

Fitzpatrick D, Usrey WM, Schofield BR, Einstein G (1994) The sublaminar organization of corticogeniculate neurons in layer 6 of macaque striate cortex. Vis Neurosci 11:307-315.

Gilbert CD, Kelly JP (1975) The projections of cells in different layers of the cat's visual cortex. J Comp Neurol 163:81-106.

Gilbert CD, Wiesel TN (1979) Morphology and intracortical connections of functionally characterized neurones in the cat visual cortex. Nature 280:120-125.

Harting JK, Hall WC, Diamond IT, Martin GF (1973) Anterograde degeneration study of the superior colliculus in Tupaia glis: evidence for a subdivision between superficial and deep layers. J Comp Neurol $148: 361-386$

Hendrickson AE, Wilson JR, Ogren MP (1978) The neuroanatomical organization of pathways between the dorsal lateral geniculate nucleus and visual cortex in old world and new world primates. J Comp Neurol $182: 123-136$ 
Hendry SHC, Yoshioko T (1994) A neurochemically distinct third channel in the macaque dorsal lateral geniculate nucleus. Science 264:575-577.

I Ioldefer RN, Norton TT (1995) Laminar organization of receptive ficld properties in the dorsal lateral geniculate nucleus of the tree shrew (Tupaia glis belangeri). J Comp Neurol 358:401-413.

Hubel DH (1975) An autoradiographic study of the retino-cortical projections in the tree shrew (Tupaia glis). Brain Res 96:41-50.

Hubel DH, Wiesel TN (1968) Receptive fields and functional architecture of monkey striate cortex. J Physiol (Lond) 195:215-243.

Hubel DH, Wiesel TN (1972) Laminar and columnar distribution of geniculo-cortical fibers in the macaque monkey. J Comp Neurol 146:421-450.

Hubel DH, Wiesel TN (1977) Functional architecture of macaque monkey visual cortex. Proc R Soc Lond [Biol] 198:1-59.

Humphrey AL, Sur M, Uhlrich DJ, Sherman SM (1985) Projection patterns of individual $\mathrm{X}$ - and $\mathrm{Y}$-cell axons from the lateral geniculate nucleus to cortical area 17 in the cat. J Comp Ncurol 233:159-189.

Kaplan E, Shapley R (1982) X and Y cells in the lateral geniculate nucleus of macaque monkeys. J Physiol (Lond) 330:125-143.

Katz LC (1987) Local circuitry of identified projection neurons in cat visual cortex brain slices. J Neurosci 7:1223-1249.

Kretz R, Rager G, Norton TT (1986) Laminar organization of ON and OFF regions and ocular dominance in the tree shrew (Tupaia belangeri). J Comp Neurol 251:135-145.

Krieg WJS (1946) Connections of the cerebral cortex. I. The albino rat: a topography of the cortical areas. J Comp Neurol 113:221-275.

Lachica EA, Casagrande VA (1992) Direct W-like geniculate projections to the cytochrome oxidase (CO) blobs in primate visual cortex: axon morphology. J Comp Neurol 319:141-158.

LeVay S, McConnell SK (1982) On and off layers in the lateral geniculate nucleus of the mink. Nature 300:350-351.

LeVay S, Sherk H (1981) The visual claustrum of the cat. I. Structure and connections. J Neurosci 1:956-980.

Lin CS, Kaas JH (1977) Projections from cortical visual areas 17, 18, and MT onto the dorsal lateral geniculate nucleus in owl monkeys. J Comp Neurol 173:457-474.

Lund JS, Boothe R (1975) Interlaminar connections and pyramidal neuron organization in the visual cortex, area 17 , of the macaque monkey. J Comp Neurol 159:305-334.

Lund JS, Lund RD, Hendrickson AE, Bunt AH, Fuchs AF (1975) The origin of efferent pathways from the primary visual cortex, area 17, of the macaque monkey. J Comp Neurol 164:287-304.

Mesulam M-M (1977) Differential sensitivity between blue and brown reaction products of HRP neurohistochemistry. Neurosci Lett 5:7-14.

Muly EC (1992) The laminar organization of intrinsic circuits in tree shrew striate cortex. $\mathrm{PhD}$ thesis, Duke University.

Muly EC, Fitzpatrick D (1992) The morphological basis for binocular and ON-OFF-convergence in tree shrew striate cortex. J Neurosci $12: 1319-1334$
Murphy PC, Sillito AM (1994) Morphology of single corticofugal axons from area 17 of cat visual cortex to the lateral geniculate nucleus (dLGN). Soc Neurosci Abstr 20:135.

Norton TT (1982) Geniculate and extrageniculate visual systems in the tree shrew. In: Changing concepts of the nervous system (Morrison AR, Strick PL, eds), pp 377-409. New York: Academic.

O'Leary JL (1941) Structure of the area striata of the cat. J Comp Neurol 75:131-164.

Raczkowski D, Fitzpatrick D (1990) Terminal arbors of individual, physiologically identified geniculocortical axons in the tree shrew's striate cortex. J Comp Neurol 302:500-514.

Schiller PH (1992) The On and Off channels of the visual system. Trends Neurosci 15:86-92.

Schiller PH, Logothetis NK (1990) The color-opponent and broadband channels of the primate visual system. Trends Neurosei 13:392-398.

Schiller PH, Malpeli JG (1978) Functional specificity of lateral geniculate nucleus laminae of the rhesus monkey. J Neurophysiol 41:788-797.

Shapley R, Perry VH (1986) Cat and monkey retinal ganglion cells and their visual functional roles. Trends Neurosci 9:229-235.

Sherman SM (1985) Functional organization of the W-, X-, and Y-cell pathways in the cat: a review and hypothesis. In: Progress in psychobiology and physiological psychology, Vol 11 (Sprague JM, Epstein AN, eds), pp 233-314. New York: Academic.

Sherman SM, Wilson JR, Kaas JH, Webb SV (1976) X- and Y-cells in the lateral geniculate nucleus of the owl monkey (Aotus trivirgatus). Science 192:475-476.

Sillito AM, Jones HE, Gerstein GL, West DC (1994) Feature-linked synchronization of thalamic relay cell firing induced by feedback from the visual cortex. Nature 369:479-482.

Stryker MP, Zahs KR (1983) On and Off sublaminae in the latera geniculate nucleus of the ferret. J Neurosci 3:1943-1951.

Sugita N (1917) Comparative studies on the growth of the cerebral cortex. II. On the increase in the thickness of the cerebral cortex during the postnatal growth of the brain: albino rat. J Comp Neurol 28:511-591.

Symonds LL, Kaas JH (1978) Connections of striate cortex in the prosimian Galago senegalensis. J Comp Neurol 181:477-512.

Usrey WM, Muly EC, Fitzpatrick D (1992) Lateral geniculate projections to the superficial layers of visual cortex in the tree shrew. J Comp Neurol 319:159-171.

Wilson JR, Hendrickson AE (1981) Neuronal and synaptic structure of the dorsal lateral geniculate nucleus in normal and monocularly deprived Macaca monkeys. J Comp Neurol 197:517-539.

Wilson PD, Rowe MH, Stone J (1976) Properties of relay cells in the cat's lateral geniculate nucleus: a comparison of W-cells with $\mathrm{X}$ - and Y-cells. J Neurophysiol 39:1193-1209.

Wiser AK, Callaway EM (1994) Local circuits in macaque V1: projections of individual layer 6 neurons. Soc Neurosci Abstr 20:310. 